\title{
基于分子间作用力的敏感效应与痕量检测生化 传感器
}

\author{
李昕欣* \\ 中国科学院上海微系统与信息技术研究所传感技术国家重点实验室, 上海 200050 \\ *E-mail: xxli@mail.sim.ac.cn \\ 收稿日期: 2015-01-25; 接受日期: 2015-04-17 \\ 国家自然科学基金(批准号: 91323304, 91023046, 61161120322)资助项目
}

\begin{abstract}
摘要在微纳米尺度的机电敏感结构表面进行功能分子层修饰, 通过与目标靶分子特异 性结合, 在表面形成 Gibbs 自由能的变化, 由此产生的纳机械表面应力可被结构上集成的机 电敏感元件转换成实时电信号输出. 首先对固体表面分子层自组装产生纳机械表面应力的 机制进行研究, 将表面上形成的单分子层(self-assembled monolayer, SAM)按作用原理在纵向 (即分子层厚度方向)上分为头基、分子链和尾基三层结构分别进行了基于纳机械敏感实验的 原理揭示, 在此基础上发明了一种作图法来定量评估和分析自组装分子层对表面能变化的 作用. 为使分子作用产生纳机械敏感效应在痕量生化分子快速检测识别中得到应用, 首次将 纵向分子特异性作用和相邻分子间横向作用区分开来, 通过不同类型分子间作用的分析和 实验验证得到如下结论：横向分子作用是产生表面应力值大小和正负(张应力或压应力)的决 定性因素, 而分子纵向作用主要是通过对分子层自组装有序性的调节来影响表面应力产生. 在对各种横向分子作用机制分析的基础上, 提出并用实验验证了分子间氢键作用可产生最 高灵敏度的纳机械敏感效应. 此后介绍了特异性分子作用产生表面应力的敏感效应在生化 痕量快速检测传感器的应用. 采用微纳悬臂梁作为敏感效应的转换器, 将表面应力转换为悬 臂梁弯曲, 利用集成在悬臂梁内的压阻器件进行电信号输出. 通过在悬臂梁表面金薄膜上修 饰巯基双层分子敏感基团, 实现了对 $\mathrm{ppb}$ 量级有机磷毒害蒸气的快速检测. 为实现敏感分子 层长期稳定工作, 针对 TNT 爆炸物分子检测提出并实现了在悬臂梁硅表面直接两次嫁接自 组装硅烷基敏感基团, 进而解决了传感器对 ppt 量级 TNT 检测的长期稳定性问题. 通过对传 感器电绝缘的有效处理, 又实现了对 $1.5 \times 10^{-11} \mathrm{~mol} / \mathrm{mL}$ 浓度链霉亲和素的生物溶液在线检测.
\end{abstract}

\section{关键词}

分子特异性作用 纳机械敏感效应 表面应力 生化传感器

\section{1 引言}

随着食品安全、环境监控以及生物医药事业的发
展，对生化分子现场快速检测识别的需求变得越来 越迫切. 而目前生化传感器能很好应用和产品化的 技术相对物理量传感器而言还是很少的. 物理类传

引用格式: 李昕欣. 基于分子间作用力的敏感效应与痕量检测生化传感器. 中国科学: 技术科学, 2015, 45: 687-704 Li X X. Sensing effects and trace-level bio/chemical sensors based on molecular interaction induced nano-mechanical force (in Chinese). Sci Sin Tech, 2015, 45: 687-704, doi: 10.1360/N092014-00361 
感器在集成电路技术和 MEMS 技术产业化大潮的推 进下, 已经在工业自动化、汽车电子和智能消费类电 子等领域获得了规模化的系列产品应用. 深入分析 生化传感器与物理传感器目前技术状态的不同, 可 以认为生化传感器比物理传感器在技术上更为复杂, 主要体现在生化传感器比物理传感器在传感机制上 多出了一个信息转换界面. 对于大多数物理类传感 器如惯性传感器、温度传感器和压力传感器, 其内部 一般都有一个将外界物理量输入引发的某种物理敏 感效应转换为可检测电信号的传感信息界面. 以悬 臂梁一质量块结构的微型加速度传感器为例, 所检 测的加速度在传感器质量块上产生的惯性力可产生 悬臂梁弯曲应力或质量块的位移, 这些物理变化可 直接转变成压阻效应下的电阻值变化或间距变化下 的电容值变化, 因此在接口电路的帮助下都能够以 电压或电流的形式直接进行电信号输出. 而对于生 化传感器而言, 其内部传感机制如图 1 所示. 除了具 有与物理传感器一样的从物理敏感效应向电信号转 换的第二界面外, 生化传感器的敏感机制中还多出 了一个界面, 可称之为第一界面. 在第一界面上, 生 化传感器要将分子特异性结合/反应(如免疫反应、酶 作用、DNA 杂交、分子吸附等)先转换为某种物理敏 感效应(如电流、热量、质量变化、机械力学响应、 光产生等), 此后才能在第二界面上再转变成电信号 输出. 与相对更加成熟的第二界面技术相比, 第一界 面上的核心问题是特异性分子作用的精确可靠表征 问题. 在纷杂环境下, 对痕量的分子特异性作用向物 理敏感效应进行快速的敏感信息的可靠转换, 确实
是一个科学难题. 为了解决该第一界面的问题, 很多 科学研究都在寻找各种分子作用表征方法来实现和 完善该第一界面的敏感表征. 本论文主要叙述微纳 结构固体表面上分子特异性作用所产生的纳机械 (nano-mechanical)敏感效应和机制. 由于纳机械敏感 效应易于被集成和固化在微纳机电系统技术形成的 微纳传感器结构所表征, 进而可以将前述的第一界 面和第二界面很好地结合起来, 因此近期被广泛关 注和寄予希望.

对表面进行纳米表征和分子成像的原子力显微 镜(AFM) 和扫描探针显微镜(SPM) 是国际公认的成功 纳机械表征技术 ${ }^{[1,2]}$. 借助微纳机械悬臂梁结构对纳 米针尖与表面分子的力的作用, 将产生的悬臂梁力 学弯曲用激光束的偏转进行表征, 实现了原子级可 分辨的探测能力. 针对纳机械应力的敏感效应表征, 如果把 AFM 的激光测量方法用在硅悬臂梁上集成的 压阻敏感器件来代替, 就可以实现直接的电学信号 输出 ${ }^{[3]}$. 而将纳米针尖对表面原子/分子的痕量力作 用转换成分子特异性作用在悬臂梁上引起的力学敏 感效应, 则可以模拟 AFM 的方式来设计开发生化微 纳传感器.

SPM(包括 AFMAFM 等)利用纳机械探针进行表 面纳米特性扫描, 主要有两种工作模式, 一种为静态 接触模式, 另一种为动态敲击模式. 而同样, 对界面 作用下的生化分子进行纳机电敏感探测, 也存在与 此相似的两种模式，即静态表面应力表征模式和动 态频率检测模式 ${ }^{[4,5]}$. 科学家们在对 SPM 探测研究过 程中发现了很多环境因素引入的信号干扰, 经过

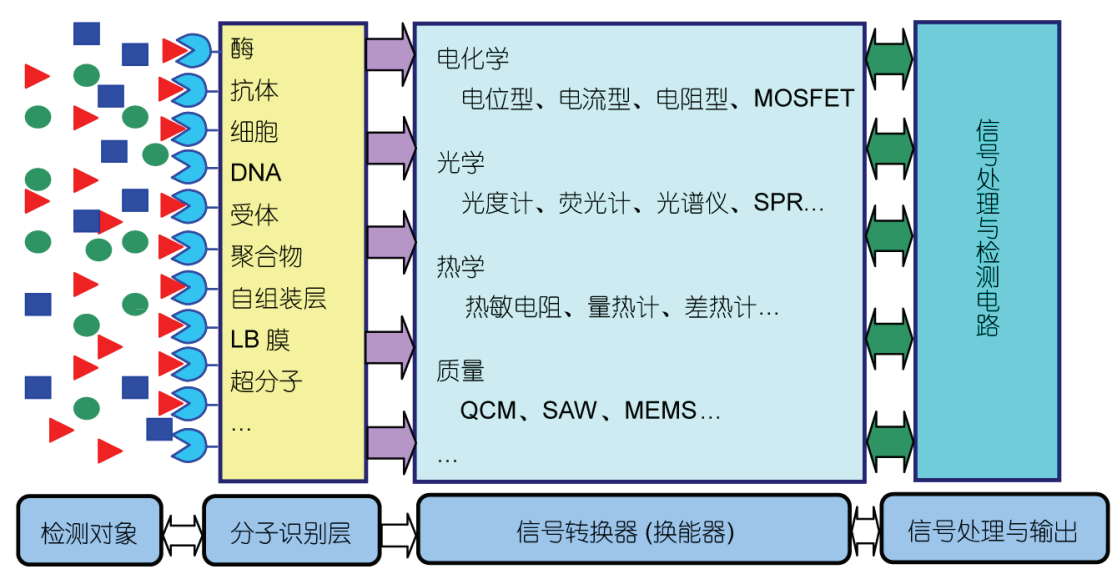

图 1 (网络版彩图)生化传感器原理性结构示意图

其内部包含从生化分子作用向物理敏感效应转换和从物理效应向电信号转换这两个信息的转换界面 
反向思考确认了微纳悬臂梁也是一个对表面分子作 用极为敏感的表征载体. IBM 和美国橡树岭国家实验 室等研究机构发现固体表面的分子层自组装或界面 分子间特异性作用可以产生纳机械表面应力, 进而 用微纳悬臂梁的弯曲实现了敏感表征 ${ }^{[6 \sim 10]}$. 同样利用 敏感悬臂梁对特异性作用对被测物分子的俘获, 通 过探测有限个数分子在悬臂梁上的质量增加, 用谐 振频率变化信号实现了 $10^{-18} \mathrm{~g}(\mathrm{ag})$ 甚至是 $10^{-21} \mathrm{~g}(\mathrm{zg})$ 的痕量检测 ${ }^{[11 ~ 15]}$. 通过科学家对该动态敏感机制检 测极限的分析, 提出了在近期去实现对单个碳原子 质量 (1 个道尔顿) 量级测量的目标 ${ }^{[16]}$.

对于纳机械静态敏感效应和基于微纳悬臂梁的 表征方法, 虽然国际上很多研究机构都在研究中采 用, 但对于其敏感机制, 始终没有一个完善的敏感效 应模型. 以往一些研究, 通常只是就具体实验中发现 的现象进行个体现象分析 ${ }^{[17,18]}$, 研究目标较为分散, 在对界面分子作用产生纳机械信号的本源探讨方面 还存在一定的片面性, 还较难对整个敏感机制和模 型进行全面阐述, 因此需要进一步深入研究. 本文将 力图对分子界面作用引起的纳机械敏感效应、机制和 传感器构筑进行详细的论述.

\section{2 产生纳机械表面应力敏感效应的 3 层分 子作用能量模型}

固体表面上自组装的 SAM 目前主要包括在金 (111)表面上自组装颈基化合物分子和在硅/氧化硅表 面自组装硅烷基化合物分子这两大类, 主要目的是 在固体表面形成功能化如表面钝化、特异性敏感和吸 附等, 也可为进一步固定生化分子提供中间层的交
联作用. 分子自组装是一种自发的纳米构筑行为, 自 组装的过程自然伴随着表面(界面)Gibbs 自由能的变 化. 很长时间以来, 对分子层自组装过程中表面能量 变化规律缺乏实验表征方法和定量化的模型. 其实 表面自由能的变化可以产生表面应力或释放已有的 表面应力, 它们的物理量纲 $\mathrm{N} / \mathrm{m}$ 与 $\mathrm{J} / \mathrm{m}^{2}$ 其实也是完 全相同的. 将自组装的表面放置在一个微米或纳米 厚度的悬臂梁上, 所产生的纳机械表面应力会导致 悬臂梁产生微弱的弯曲. 该弯曲与表面应力的关系 可以用如下的 Stoney 方程 ${ }^{[19]}$ 来表示:

$$
\frac{1}{R}=\frac{6(1-v)}{E t^{2}} \sigma,
$$

其中 $R, t, v, E$ 和 $\sigma$ 分别为悬臂梁弯曲曲率半径、厚度、 材料泊松比、杨氏模量和表面应力. 除了可以像 AFM 一样用光束的偏转来检测该表面应力引起的弯曲外, 本研究组研制的 $1 \mu \mathrm{m}$ 厚氧化硅悬臂梁在内部集成了 被氧化层绝缘体包裹的单晶硅压阻敏感薄层 ${ }^{[20]}$, 可 以将表面应力引起悬臂梁弯曲转变成悬臂梁体弯曲 应力用压阻效应进行直接的电学输出表征 ${ }^{[21]}$. 氧 化硅具有比硅更低的杨氏模量即更高的力学弯曲灵 敏度, 而氧化硅包裹的硅压阻元件比传统 PN 结隔离 的压阻元件具有更低的噪声效应即更高的传感信 噪比.

只有实验的表征手段还远远不够. 如果还是将 表面分子层自组装引起的效应看成是发生在无限薄 (即没有厚度)的界面上, 从纳米尺度下的微观角度来 看是不科学的. 以颈基化合物分子在金表面上自组 装为例, 如图 2 所示, 将该分子层自组装的界面从厚 度上分为固体表面/头基层、分子链层和尾基/环境层 这样 3 个相对独立且有代表性的层次. 根据不同层的
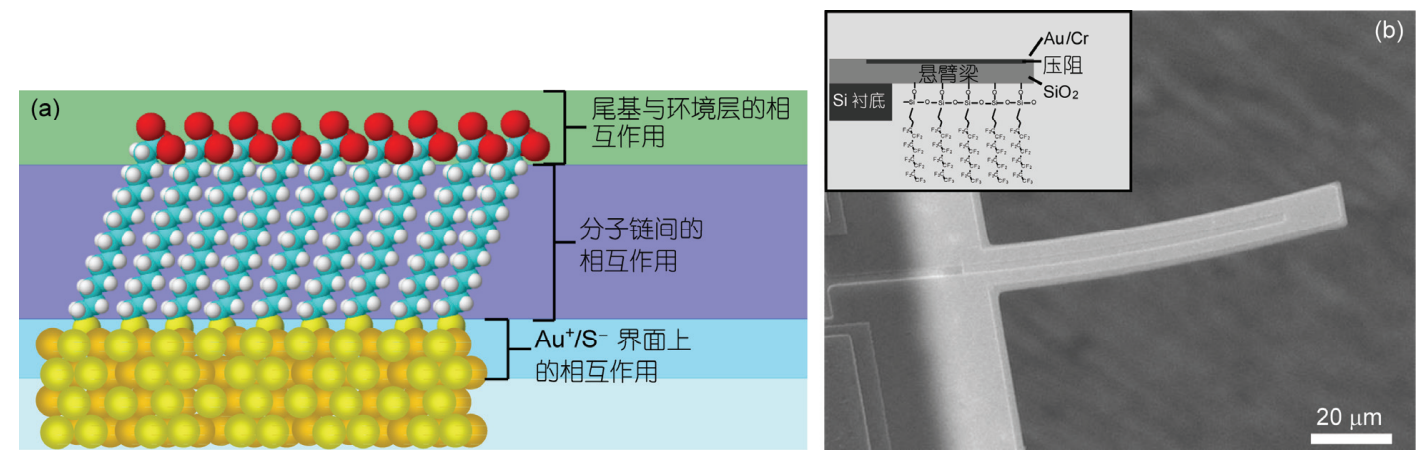

图 2 (网络版彩图) (a) $\mathrm{Au}\left(111\right.$ ) 表面自组装 SAM 的 3 层相互作用模型示意图; (b) $\mathrm{SiO}_{2}$ 微悬臂梁的扫描电子显微镜(SEM) 图 和表面结构示意图 ${ }^{[25]}$ 
结构特点, 我们的结构模型中保留了各层内相邻结 构的横向作用. 在悬臂梁上表面淀积了纳米厚度的 金，而将悬臂梁的下表面预先修饰了超低表面能的 氟硅烷分子层 FAS-17, 这样可以保证悬臂梁的弯曲 不受环境水分子吸附对悬臂梁下表面的影响.

3 个结构层对表面能变化的贡献分别来自: 1) 第 1 层上有金一硫间配位键作用, 被广泛认为是相邻电 偶极子库伦排斥引起的金晶格驰豫或表面重构 ${ }^{[22 ~ 24]}$; 2) 第二层上有相邻分子链间的作用, 还包括了平衡 自组装间隙与金晶格尺度间差异所造成的体位作用, 因此有限链长的作用被认为与链长成正比; 3) 尾基 与环境间的作用, 其值可以用表面接触角测量实验 来直接获得. 3 个次作用相互叠加后, 总的作用对界 面能变化的贡献可以表达为

$$
\Delta G=\left(\Delta G_{\mathrm{Au}-\text { initial }}+\Delta G_{\mathrm{Au}-\mathrm{S}}\right)+\Delta G_{c}+\Delta G_{t},
$$

$\Delta G_{\mathrm{Au} \text {-initial }}$ 是暴露在自组装介质中的金表面能量与纯 净金表面能量的差值, $\Delta G_{\mathrm{Au}-\mathrm{S}}$ 是 $\mathrm{Au}-\mathrm{S}$ 键导致的界面 自由能变化, $\Delta G_{c}$ 体现分子链间作用的贡献, 而 $\Delta G_{t}$ 是尾基与环境作用的贡献. 与表面自由能变化相对 应, 分子自组装界面上产生的等效表面应力也可相 应进行三个层面的分解而表达为

$$
\Delta \sigma=\left(\Delta \sigma_{\mathrm{Au}-\text {-initial }}+\Delta \sigma_{\mathrm{Au}-\mathrm{S}}\right)+\Delta \sigma_{c}+\Delta \sigma_{t},
$$

式中每层作用对表面应力的贡献在数值上等于其对 总界面自由能变化的贡献值. 为了显示出不同层结 构独特的作用效果, 我们在表征自组装导致悬臂梁 表面应力的实验中专门选取了如下四种颈基化合物 分子: 十二烷基硫醇(DDT)、十六烷基硫醇(HDT)、
十七氟癸基硫醇(FAT-17)和 3-颈基丙酸(3-MPA), 这 些分子均含有一个共同的 $-\mathrm{CH}_{2} \mathrm{CH}_{2} \mathrm{SH}$ 结构. 由于相 隔 2 个亚甲基，可以认为连接在这个结构上的分子链 对硫原子产生的电子效应可以忽略, 因此在自组装 过程中, Au-S 键结合界面层的作用对总界面能变化 的贡献值相同. 所以，这些化合物分子自组装产生的 界面自由能变化的大小主要取决于 $\mathrm{Au}$ 的初始界面自 由能能、分子链间的作用以及末端基与周围介质的作 用.

采用图 2 中所示的本实验室研制的集成压阻微 悬臂梁，在悬臂梁上表面的金薄膜上对四种颈基化 合物分子分别进行自组装与表面应力实时在线记录, 而所有的自组装实验又分别在极性溶剂乙醇和乙腈、 非极性溶剂正已烷以及空气等四种反应介质中顺序 进行. 图 3 是悬臂梁传感器记录的所有检测结果. 通 过实验分析和分子间结构对比，我们得到了如下几 点重要的结论.

1) 自组装介质对初始界面能的影响很大. 在不 同的介质中生长颈基化合物 SAM，尽管最终形成 SAM 的结构完全相同，但是由于其所处的介质环境 不同，其所产生的界面能的变化量就大不相同. 而初 始界面能是自组装反应的能量变化起点, 对 SAM 形 成过程中产生的界面能变化非常重要. 这些巯基化 合物在乙醇介质中自组装产生界面能变化的顺序与 在空气中的完全相同，只是响应值都比在空气中更 大, 从图中数据对比可以看出, 同一化合物在这两种 不同介质中自组装导致的界面能变化的差值都在 0.6 $\mathrm{J} / \mathrm{m}^{2}$ 左右, 这主要是由于 $\mathrm{Au}$ 的初始界面能不同所致.
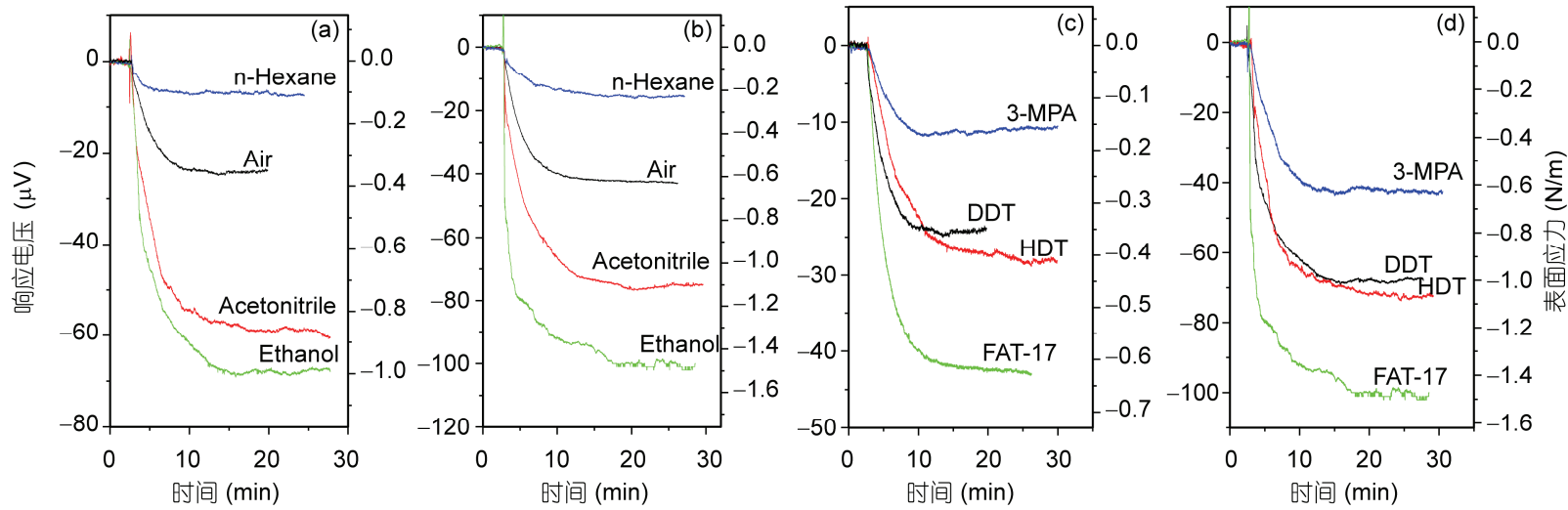

图 3 (网络版彩图)悬臂梁传感器测量自组装过程的响应曲线

(a)和(b)分别是 DDT 和 FAT-17 在四种不同介质中的自组装结果; (c)和(d)分别是在空气和乙醇介质中四种颈基化合物自组装引起的传感器 响应 ${ }^{[25]}$ 
由此我们认为, 颈基化合物在 $\mathrm{Au}$ 表面结合后, 形成 了 $\mathrm{Au}^{+}-\mathrm{S}^{-}$界面层, 从而改变了 $\mathrm{Au}$ 表面上的原子间相 互作用能的大小, 这可以很大程度地降低 $\mathrm{Au}$ 的表面 能. 因此导致表面能降低多少, 这很大程度上取决于 $\mathrm{Au}$ 的初始界面能, $\mathrm{Au}$ 的初始界面能越大, 其在自组 装过程中降低的趋势就越大, 由自组装导致的界面 能改变也越大.

2) 对于不同颈基化合物在同一介质中的自组装 过程, 方程(2)中括号内的两项可以被认为是相同的, 其不同之处主要在于烷基链之间的作用以及末端基 与环境介质间的作用对界面能变化带来的贡献. 从 实验结果中可以看出, HDT 自组装产生的响应值比 DDT 略大, 两种烷基硫醇的响应值要小于氟烷基硫 醇 FAT-17 但大于 3-MPA. 这说明颈基化合物的分子 链间作用对界面能变化的贡献十分显著, 该结果与 Berger 等人 ${ }^{[6]}$ 和 Desikan 等人 ${ }^{[22]}$ 所观察到的实验现象 相吻合. 而对于氟烷基硫醇来说, 由于氟原子的直径 比氢大, 其分子链间的平衡距离也大于烷基硫醇, 因 而对于相同长度的分子碳链来说, 由氟烷基分子链 间相互作用导致的表面能变化要大于一般烷基链间 的作用. 从图 4 的实验结果可以清楚看到这一点. 对 有限链长的同种分子来说, 其链长部分对表面能变 化的贡献是基本呈线性关系的, 图 4 中烷基硫醇链长 作用已由经过 3 种链长 (分别为 3,12 和 14 个碳链) 烷 基硫醇实验点所画的直线来表示. 而对应于链长为 10 的地方, 氟烷基硫醇的链长作用明显比普通烷基 的要大很多.

3) 末端基与环境介质之间的相互作用可以用界

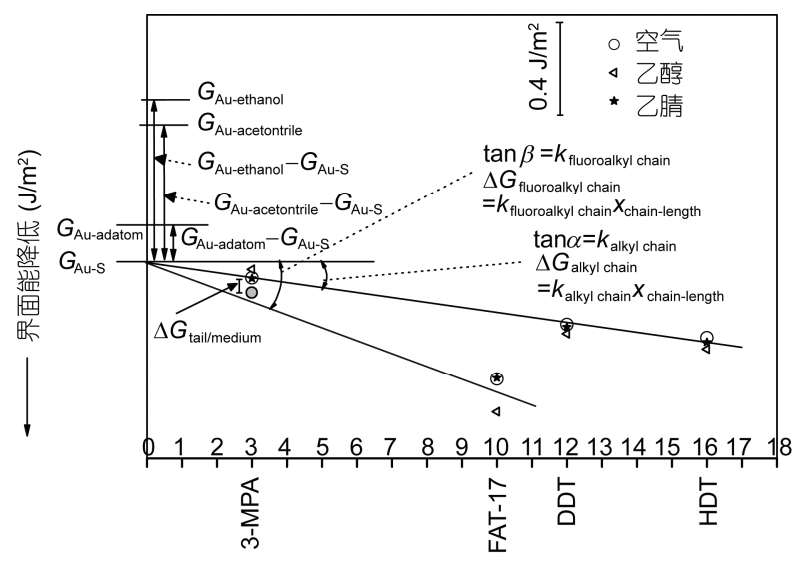

图 4 分子自组装相关的各层对界面能变化贡献的定量模 型推算示意图 ${ }^{[25]}$
面能的大小来表征. 基于水和十六烷接触角测量数 据, 用 OWRK 方法 ${ }^{[23,24]}$ 可以计算得到不同 SAM 在空 气中的表面能, 由此进一步可计算出末端基与介质 之间的界面能. 总的来说, 末端基与介质的作用对界 面能变化的贡献显然要小于链间相互作用和头基相 互作用的贡献. 无其是对于长链颈基化合物来说, 末 端基与介质作用的贡献可以忽略.

从以上实验结果可以看出 ${ }^{[25]}$, 自发的自组装过 程是由于过剩的界面自由能导致的, 这一过程通常 伴随界面自由能的下降和界面张力的释放. 这就等 价于在这个表面产生了压应力. 理想的 $\mathrm{Au}$ 表面具有 很高的表面能, 将其置于不同环境介质中时, 其表面 能将出现不同程度的下降, 成为了 $\mathrm{Au}$ 在不同介质中 的初始界面能. 当 $\mathrm{Au}$ 表面结合颈基化合物形成 SAM 后, $\mathrm{Au}$ 的表面能将下降至一个固定值 $G_{\mathrm{Au}-\mathrm{S}}$. 在此基 础上, 分子链的相互作用将导致界面能进一步下降, 分子链作用能的大小因链长和链的种类不同而改变. 此外, 末端基与介质的作用也可以一定程度上影响 界面能变化的大小. 引起界面能改变的 3 层作用可以 定量地进行模型表征, 本文利用画图法建立了该定 量表征模型，显示在图 4 中. 假设分子链间作用对界 面能变化的贡献与链长成正比, 由测量得到的总表 面能变化和末端基与介质的界面能变化数据推算, 可以得到链长为 0 时, 即 $\mathrm{Au}^{+}-\mathrm{S}^{-}$键结合后的界面能的 相对位置 $G_{\mathrm{Au}-\mathrm{S}}$, 这是颈基化合与 $\mathrm{Au}$ 结合的第 1 层. 对于研究中选用的这几种颈基化合物, 无论其初始 界面能大小如何, 头基与 $\mathrm{Au}$ 表面结合后形成 $\mathrm{Au}^{+}-\mathrm{S}^{-}$ 界面层的 $\mathrm{G}_{\mathrm{Au}-\mathrm{S}}$ 大小总是固定的. 尽管目前还没有办 法估算出 $\mathrm{G}_{\mathrm{Au}-\mathrm{S}}$ 的绝对值大小, 但是我们可以通过实 验测量得到的数据, 计算出它与初始界面能以及链 作用导致界面能变化的差值. 计算得到了 $G_{\mathrm{Au} \text {-adatom }}$ 和 $G_{\mathrm{Au}-\mathrm{S}}$ 间的差值约为 $0.16 \mathrm{~J} / \mathrm{m}^{2}, G_{\mathrm{Au}-\mathrm{air}}$ 和 $G_{\mathrm{Au}-\mathrm{S}}$ 的差 值约为 $0.76 \mathrm{~J} / \mathrm{m}^{2}$. 烷基链间作用的贡献与长度的关 系约为每节碳链 $0.015 \mathrm{~J} / \mathrm{m}^{2}$; 而氟烷基分子链间具有 更强的作用, 贡献约为每节碳链 $0.032 \mathrm{~J} / \mathrm{m}^{2}$.

我们通过建立颈基化合物自组装层在 $\mathrm{Au}$ 表面的 3 层表面能作用模型，描述了颈基化合物在 $\mathrm{Au}$ 表面 自组装导致的界面能变化, 进一步用悬臂梁测量结 果对模型进行了验证, 并基本定量地估算出每层作用 对总界面能变化的贡献 ${ }^{[25]}$. 这种作用模型可以使人 们进一步用于解释传感器的敏感响应机制, 还可以 用来设计传感器实现纳机械表面应力灵敏度的优化. 


\section{3 分子间横向作用产生纳机械敏感信号的 机制分析}

我们的很多实验结果都表明，垂直于界面的纵 向分子作用并不是影响悬臂梁表面应力产生的唯一 因素. 当被检测分子吸附于固体表面时, 相邻的分子 间将产生各种相互作用, 以达到能量最低的平衡状 态. 也可以这样说, 基于分子层间特异性的纵向作用 (即敏感材料分子层对被感知的分子产生的吸附作 用), 分子结合特异性的高低对结合速度和吸附分子 表面覆盖率有最大的影响. 但该纵向作用对界面上 产生纳机械敏感信号大小的直接贡献并不大, 其更 重要的功能是借助特异性快速形成致密而有序的分 子吸附层. 而在纵向作用帮助下, 致密有序分子层形 成后会产生相邻分子间的横向相互作用(图 5). 虽然 在上一节中我们简单讨论了分子链间横向作用, 但 那里实验所采用的分子都是横向作用相对较弱的, 体现的纳机械敏感效应与头基界面层相比而言并不 显著. 基于更多的实验和作用机理分析, 本文深入研 究了界面上被吸附分子间横向作用对表面应力产生 的影响原理，讨论了包括空间体位作用、范德华力、 静电力和氢键作用等分子间横向作用对产生表面应 力效应的机制 ${ }^{[26]}$.

\section{1 空间体位作用}

空间体位作用是可以显著影响特异性吸附所产 生表面应力大小的一个重要因素, 在某些情况下, 甚 至可以决定所产生表面应力的方向即产生张应力或 压应力. 据报道, 2 个配对的单链 DNA(ssDNA) 杂交 所导致的构型熵变化可实现对静态微悬臂梁产生弯 曲方向的控制 ${ }^{[27]}$. 除了 DNA 分子复杂之外, 我们在 实验中还发现, 爆炸物 TNT 分子在吸附于结构敏感 层上后也可以产生方向相反的表面应力. 我们实验 中分别使用 4-颈基苯甲酸(4-MBA)修饰在金表面作

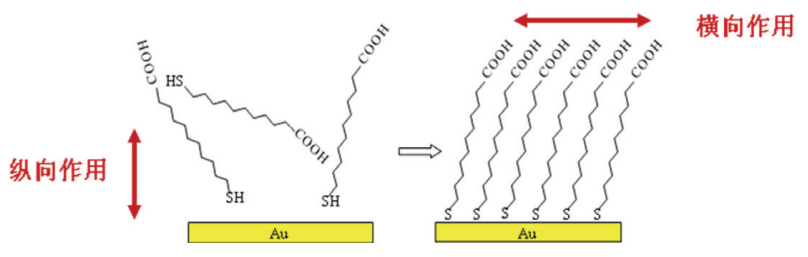

图 5 (网络版彩图)固体表面上分子层形成过程中分子特异 性纵向作用和分子间横向作用的示意
为敏感层以及末端基为 $-\mathrm{ph}-\mathrm{COOH}$ 的双分子层修饰 在二氧化硅表面作为敏感层，对同一浓度的 TNT 气 体分子进行了检测，实验所得相反方向的悬臂梁表 面应力响应结果如图 6 所示 ${ }^{[26]}$. 值得注意的是, 虽然 在本试验中悬臂梁均为向下弯曲，但是金是在悬臂 梁的上表面, 所以产生的表面应力为压应力，而 $\mathrm{SiO}_{2}$ 是在悬臂梁的下表面，所以产生的表面应力为张应 力. 由此, 两组对比实验所产生的表面应力方向是截 然相反的.

对于间硝基芳香化合物(如 TNT 或 DNT 等)来说, 苯环的作用使得两个相邻硝基的位置相对固定，用 Gaussian03 软件计算两个相邻硝基上最临近氧原子 的距离为 4.92 5.03 $\AA$. 被检测的 TNT 分子与甲基比 (a)

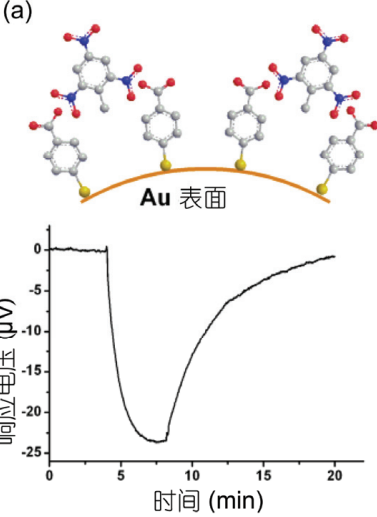

(c)

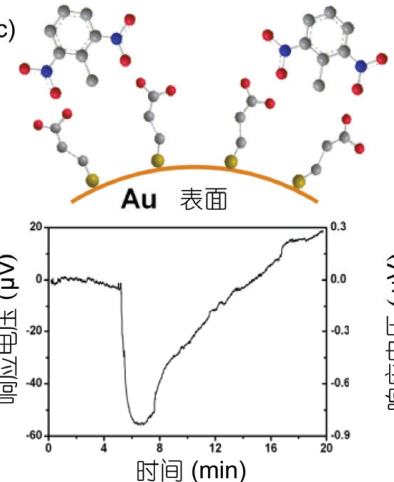

(b)

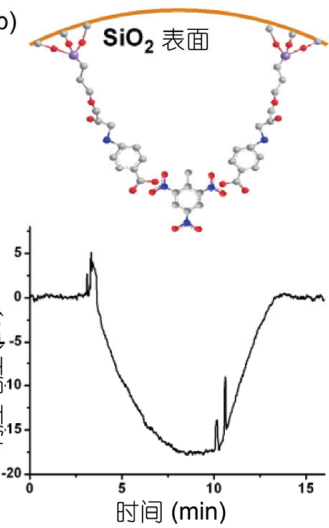

(d)

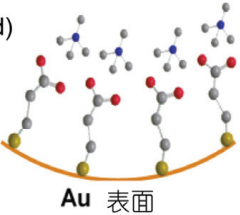

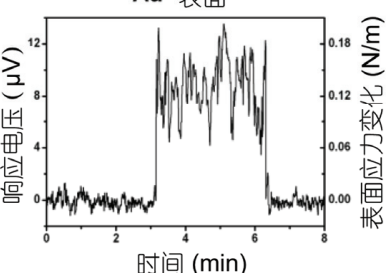

图 6 (网络版彩图) (a) TNT 在修饰金表面的微悬臂梁器 件上的吸附产生压应力; (b) TNT 在修饰 $\mathrm{SiO}_{2}$ 表面的微悬 臂梁器件上的吸附产生张应力; (c) DNT 在微悬臂梁器件 上的吸附产生压应力; (d) TMAH 在微悬臂梁器件上的吸 附产生张应力 ${ }^{[26]}$

(a)和 (b) 是不同敏感层对 TNT 蒸气的器件响应结果, 显示出相反 方向表面应力; (c)和(d)的结果显示出同一敏感层对不同尺寸的分 子的响应产生了方向相反的表面应力 
邻的 2 个硝基上最近氧原子的优化距离为 $5.2 \AA$. 当 特异性吸附反应发生时, 1 个 TNT 分子恰好可以结合 敏感层上 2 个相邻的- $\mathrm{COOH}$ 基团, 这种结合显现了 较好的立体方位选择性.

作为敏感层的 6-MNA SAM, 2 个相邻分子上的 $-\mathrm{COOH}$ 的距离与(111)面上 2 个 $\mathrm{Au}$ 原子的距离同样

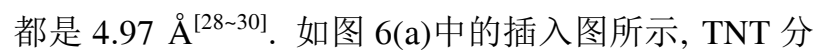
子上 2 个相邻的硝基可以与表面上 2 个相邻的 $-\mathrm{COOH}$ 结合, 形成图中所示的结构. 由于 $\mathrm{Au}$ 表面上 固定的颈基敏感分子之间的距离小于吸附了 TNT 分 子之后的平衡距离, 因此产生了向下的压应力.

对于硅氧烷双分子层而言, 首先, 必须考虑 $\mathrm{SiO}_{2}$ 表面羟基的密度对于硅氧烷自组装的影响. 在 $\mathrm{SiO}_{2}$ 表面进行硅氧烷分子自组装的化学反应为

$$
\begin{aligned}
& \equiv \mathrm{Si}-\mathrm{OH}+\left(\mathrm{H}_{3} \mathrm{CO}\right)_{3}-\mathrm{Si}-\mathrm{R} \\
& \rightarrow \equiv \mathrm{Si}-\mathrm{O}-\mathrm{Si}-\mathrm{R}+\mathrm{CH}_{3} \mathrm{OH}
\end{aligned}
$$

我们认为, $\mathrm{SiO}_{2}$ 表面的硅羟基 $(\equiv \mathrm{Si}-\mathrm{OH})$ 密度对于 硅氧烷分子自组装程度具有决定性的影响. 根据文 献[31], 在不考虑表面缺陷并认为硅氧烷分子理想致 密组装的情况下, 理论计算得到的 $\mathrm{SiO}_{2}$ 表面自组装 硅氧烷化合物分子的最大密度为每平方米 $5 \times 10^{18}$ 个 分子. 由此可以计算得出表面上相邻的 2 个硅氧烷分 子之间距离的最小值为 $d=0.44 \mathrm{~nm}$. 本实验中 ${ }^{[26]}$, 在 $\mathrm{SiO}_{2}$ 表面进行第 1 层硅氧烷分子自组装之前, 虽然用 Piranha 溶液对表面进行了羟基增强处理, 但实际操 作中 $\mathrm{SiO}_{2}$ 表面的羟基密度必然大大低于理论计算值. 也就是说, 在第 1 层硅氧烷分子自组装之前, $\mathrm{SiO}_{2}$ 表 面两相邻硅羟基之间的距离要大于 $0.44 \mathrm{~nm}$. 其次, 在第 1 层硅氧烷分子自组装反应的无水条件下, $\mathrm{SiO}_{2}$ 表面 2 个相邻硅羟基间会自发地进行缩聚反应而形 成表面惰性位点 ${ }^{[32]}$. 在惰性位点处硅氧烷分子则无 法进行自组装, 导致相邻 2 个硅羟基之间的距离甚至 达到原来的 3 倍, 从而导致硅羟基密度进一步降低. 基于上述两方面的原因, 当用硅氧烷敏感双层膜修 饰的悬臂梁检测 TNT 爆炸物蒸气时, 相邻的两个敏 感双层膜的羧基(-COOH) 将吸附 1 个 TNT 分子上的 2 个硝基 $\left(-\mathrm{NO}_{2}\right)$ 并形成氢键. 由于相邻 2 个- $\mathrm{COOH}$ 之 间的距离要远大于 2 个 $-\mathrm{NO}_{2}$ 之间的距离, 分子之间 的相互作用会产生相互吸引的作用力, 从而产生表 面张应力, 导致悬臂梁向下弯曲, 情形如图 6(b)中的 插入图所示.

本实验中所用的两组敏感层, 其用以特异性捕
捉 TNT 分子的末端基团均为- $\mathrm{Ph}-\mathrm{COOH}$, 我们可以 近似地认为, 两组敏感层与 TNT 分子发生特异性结 合的强度是相同的. 但由于不同的体位作用所产生 的巨大差别, 最终使 TNT 在两组敏感层上发生吸附 时分别产生了相反方向的表面应力信号 ${ }^{[26]}$.

换一个角度进行实验，当使用同一种敏感层检 测不同的被测分子时, 由于被吸附分子尺寸上的巨 大差别, 也可以导致产生方向相反的表面应力信号. 本文使用了三統基丙酸 (3-MPA)修饰的悬臂梁器件 来检测爆炸物 DNT(2,4-二硝基甲苯)和 MEMS 界广泛 使用的硅微机械腐蚀液四甲基氢氧化铵(TMAH)这两 种不同大小的目标分子. 实验所得相反方向的悬臂 梁表面应力响应结果如图 6(c)和(d)所示. 如前所述, 金(111)面上生长的颈基化合物之间的平衡距离为 $4.97 \AA$, 而 DNT 分子中的最大间距为 $7.04 \AA$ 分, TMAH 分子中的最大间距却为 $4.22 \AA$. 对于相同的悬臂梁传 感器, 不同的目标分子与悬臂梁上相同的敏感层分 子特异性结合, 产生了不同方向的表面应力, 使得悬 臂梁朝着不同的方向发生微纳机械弯曲. 具体地说, 具有更小尺寸的 TMAH 分子在悬臂梁表面产生了张 应力纳机械信号, 使得悬臂梁向上弯曲; 相反地, 分 子尺寸相对更大的 DNT 分子在悬臂梁表面的特异性 吸附却产生了压应力, 使得悬臂梁向下弯曲.

\section{2 范德华力作用}

我们进行的分子自组装实验表明, 在空气中 DDT 和 HDT 向金表面的自组装, 所产生的表面应力 在大小上存在一定差别. HDT 在金表面自组装时所 产生的表面应力要大于 DDT 在金表面自组装时所产 生的表面应力. 分析后认为这是由于范德华力的作 用而导致的差别. 范德华力在前人的文献中作为解 释分子特异性吸附导致悬臂梁弯曲的机理已得到了 实验证实 ${ }^{[33]}$. 使用 Lifshitz 理论对范德华力的精确计 算需要用到复杂的多层作用模型, 并且对不同的敏 感层及吸附质分子, 需要做不同的讨论. 此处, 本文 将模型简化, 近似将分子看作为一个质点, 对 Lennard-Jones 势的表达如下:

$$
V\left(r_{i j}\right)=\frac{A}{r_{i j}^{6}}+\frac{B}{r_{i j}^{12}},
$$

其中, $r_{i j}$ 是分子 $i$ 与分子 $j$ 之间的距离, $A$ 和 $B$ 是 Lennard-Jones 常数. 根据文献[33]中的计算, 由于范 德华力而导致的表面应力变化可以表达为 


$$
\begin{aligned}
\Delta \sigma_{v d w}= & \frac{3 Q}{r^{7}}\left[2 A_{2}+\frac{A_{1}}{\left[1 / 4+(d / r)^{2}\right]^{4}}\right] \\
& +\frac{6 Q}{r^{13}}\left[2 B_{2}+\frac{B_{1}}{\left[1 / 4+(d / r)^{2}\right]^{7}}\right],
\end{aligned}
$$

其中 $Q$ 是表面应力引起悬臂梁弯曲前的数密度 (number per width). 经过分析可以看出, 作为一个分 子间的短程作用力, 与其他引起表面应力变化的因 素相比较, 范德华力对总体表面应力变化的贡献不 是很大. 以烷烃颈基化合物在金表面的自组装为例, 烷基链之间的范德华力总的来说对产生表面应力值 的贡献虽不大, 但它将导致烷基链自组装层产生一 个与法线方向的倾斜角, 对产生平衡有序的表面分 子链排布十分有利 ${ }^{[17]}$.

\section{3 静电力作用}

静电力作用也被提出作为一种解释特异性分子 吸附导致悬臂梁弯曲的机理, 尤其是解释系统中存 在带电荷离子或电子转移过程的情况. 从微观角度 进行计算, 根据文献[33], 该种力产生表面应力的表 达式为

$$
\Delta \sigma_{e}=\frac{Q q^{2}}{2 \pi \varepsilon_{0} r^{2}} \sum_{n=1}^{\infty} \frac{1}{n}\left[-1+\left(1+\frac{d^{2}}{r^{2} n^{2}}\right)^{-3 / 2}\right],
$$

其中, $q$ 为所带电荷电量, $\varepsilon_{0}$ 是介电常数. 在不存在带 电荷离子的大多数系统情况下, 静电力作用可以被 考虑为偶极子之间通过静电场的相互作用. 此时, 静 电作用对最终产生的表面应力数值影响不是很大. 并且由于相邻电偶极子的带点特性完全相同, 它们 间的作用力一定是排斥力, 因此所产生的表面应力 一定是压应力. 但是在吸附质为阳离子/阴离子的情 况下, 静电作用对表面应力变化将产生非常大的影 响, 且由于是相互吸引作用, 一定会产生表面张应力. Sushko 等人 ${ }^{[34]}$ 研究了相邻羧酸根离子 $\left(-\mathrm{COO}^{-}\right)$之间 的静电排斥作用所产生的表面压应力. 但在我们小 组使用羧酸根阴离子对铵根阳离子进行检测的实验 中, 正如图 7 中显示的那样, 所测正离子对两侧敏感 材料分子的负离子敏感基团静电吸引, 所以产生的 表面应力为张应力 ${ }^{[35]}$.

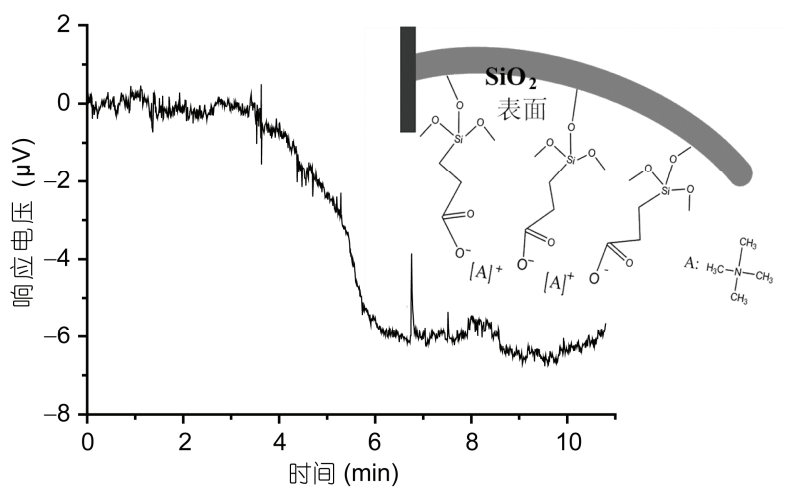

图 7 悬臂梁在 $\mathrm{TMAH}$ 水溶液溶液中对 $\left(\mathrm{CH}_{3}\right)_{4} \mathrm{~N}^{+}$离子检测 产生的表面张应力由实验测到 ${ }^{[26]}$

\section{4 分子间氢键的横向作用}

在生化检测实验中, 人们常常使用某一敏感层 对一类物质进行检测，根据敏感层与吸附质之间的 作用强度进行推断, 希望得到有规律性响应结果. 然 而, 在某些情况下, 实验结果却与预期相反, 不能用 原有的机理进行合理的解释. 我们使用了一种以羧 基为尾端基团的敏感层对一系列的胺类化合物(分别 为一甲胺、二甲胺和三甲胺)进行了检测, 并结合谐振 式微悬臂梁传感器频率信号对吸附质量的微称重 (micro-gravimetry)原理检测, 对表面应力型微悬臂梁 传感器的结果进行分析 ${ }^{[26]}$.

图 8 给出了谐振式悬臂梁传感器与应力型悬臂 梁传感器对胺类同系物的联合检测结果. 图中可以 看出, 按照甲胺、二甲胺、三甲胺的顺序来看, 随着 分子中甲基数目的增加, 谐振式微悬臂梁传感器的 测试相应幅度是依次递增的, 而应力型静态微悬臂 梁传感器的测试结果反而却是依次递减的. 根据气 相分子的碱度理论, 由于甲基具有给出电子的诱导 效应, 界面上特异性吸附反应强度顺序应与吸附质 气相碱度的大小顺序一致, 顺序为: $\mathrm{MeNH}_{2}<\mathrm{Me}_{2} \mathrm{NH}<$ $\mathrm{Me}_{3} \mathrm{~N}$. 这与质量型谐振式微悬臂梁的测试结果一致. 从图 8(a), (b)和(c)中可以看到, 谐振悬臂梁对 $1 \mathrm{ppm}$ 浓度的甲胺、二甲胺以及三甲胺气体的响应值分别为 $11.9,43$ 和 $65 \mathrm{~Hz}$. 考虑到选取的 3 种胺类同系物存在 分子量的差别 ( $M$ 甲胶: $M$ 二甲服: $M$ 三甲抆=31:45:59), 可以 进一步计算出传感器对同一浓度胺类同系物的吸附 摩尔比为 $1: 2.47: 2.85$. 然而, 应力型静态微悬臂梁 的检测信号幅度顺序却恰恰相反, 其大小顺序为 $\mathrm{MeNH}_{2}>>\mathrm{Me}_{2} \mathrm{NH}>>\mathrm{Me}_{3} \mathrm{~N}$, 从图 8 (d), (e)和(f)中可以 

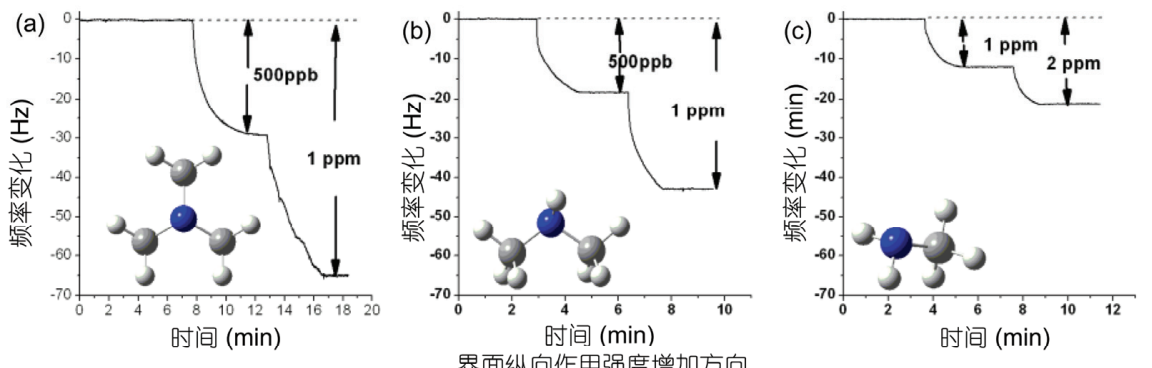

界面纵向作用强度增加方向

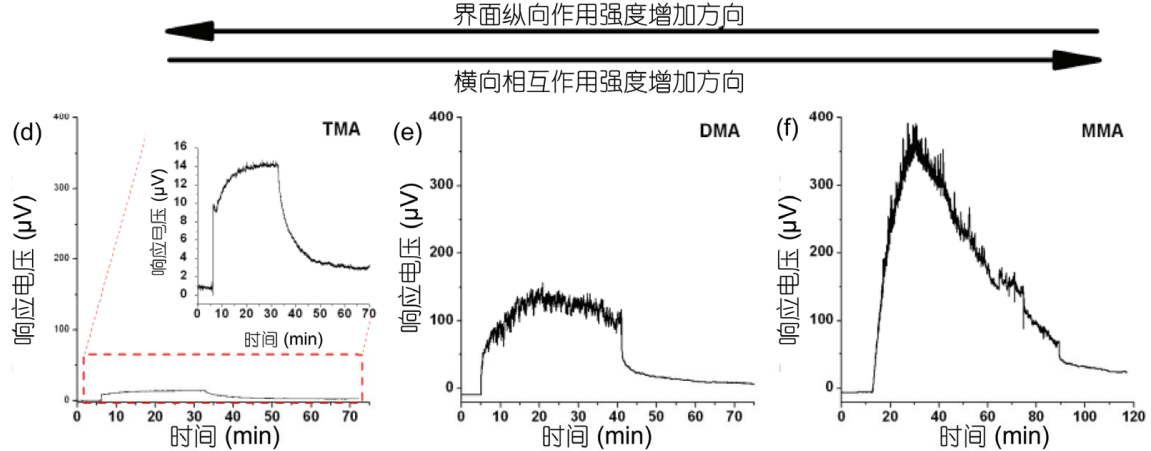

图 8 (网络版彩图)谐振式悬臂梁传感器与应力型悬臂梁传感器对胺类同系物的响应结果

(a) (c) 谐振式传感器分别对三甲胺、二甲胺和甲胺的响应; (d) (f) 应力型传感器分别对三甲胺、二甲胺和甲胺的响应 ${ }^{[26]}$

看到, 响应大小的巨大差异达到了几倍甚至一个数 量级 ${ }^{[26]}$.

这一组胺类同系物分子从结构、大小和性质上都 非常类似，对实验得到的应力型静态悬臂梁上特异 性吸附响应信号大小顺序, 用之前提到的界面纵向 作用、位阻作用及其它作用模型都无法解释. 经过分 析，我们首次使用氢键理论对其进行了解释. 胺类同 系物分子在性质上具有很大的相似性, 但其形成分 子间氢键的能力却各有不同. 如图 9 的横向分子间作 用模型所示, 三甲胺由于氮原子上连接了 3 个甲基, 没有活泼氢存在, 故不能形成分子间氢键. 二甲胺分 子中, 有 1 个甲基被氢原子所取代, 导致氮原子上所 带负电荷增加, 形成活泼氢, 可以与相邻的二甲胺分 子下面结合的敏感集团形成 1 个横向作用的分子间 氢键. 类似地, 在甲胺分子中, 有 2 个甲基被氢原子 所取代, 氮原子数所带电荷进一步增加, 与相邻甲胺 分子所形成氢键的强度也显著增加. 由于分子间氢 键的形成, 横向分子间氢键作用成为影响悬臂梁表 面应力的主要因素, 导致三甲胺与二甲胺之间敏感 响应大小上出现了数量级的差异. 由于甲胺分子间 氢键的作用强度要强于二甲胺, 甲胺分子在悬臂梁 表面的吸附将导致更大的表面应力变化, 其响应大
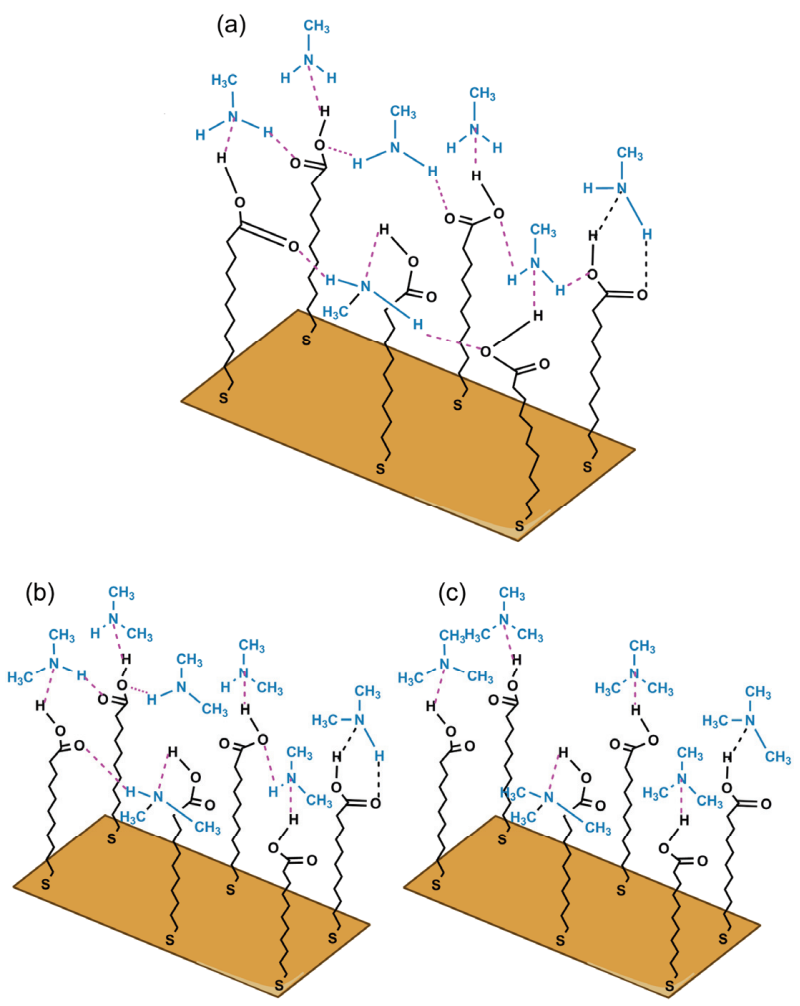

图 9 (网络版彩图)甲胺(a)、二甲胺(b) 和三甲胺(c)分子在表 面特异性吸附后横向氢键作用几何构型模型[5] 
小也达到了二甲胺的数倍.

氢键作用是一种较强的分子间力, 在许多化学/ 生化作用中, 都起着非常大的作用. 氢键的作用强度 远大于范德华力, 仅比共价键和离子键稍弱, 属于次 级键的一种, 键能在 $42 \mathrm{~kJ} / \mathrm{mol}$ 以内. 在生化传感器 的应用中, 氢键作用已被利用于作为敏感层与被吸 附分子之间的特异性反应, 许多生化传感器的敏感 层都是依靠氢键作用来捕获目标分子的. 然而, 之前 没有研究报道明确表明横向分子间氢键作用可用来 产生更大的表面应力敏感信号. 我们的实验结果表 明, 横向作用中的分子间氢键可对静态应力型微悬 臂梁生化传感器的响应可产生决定性的影响, 有无 分子间氢键形成甚至可导致高达一个数量级的表面 应力变化差别. 这为静态应力型微悬臂梁生化传感 器的发展提出了新的敏感方法和提高传感器灵敏度 设计的依据.

综上所述，分子特异性吸附导致表面应力的产 生, 是许多因素共同作用的结果. 除了界面上的纵向 作用之外, 分子间的横向作用对表面应力的产生起 着至关重要的作用. 在大部分传感器应用中, 范德华 力和静电力对表面应力的贡献不大. 而位阻作用的 影响有时可导致相反方向的表面应力. 对于位阻作 用影响不大的情况, 分子间的横向氢键作用则可以 成为引起表面应力变化最重要的作用方法, 利用该 作用可以设计出高灵敏效应的传感器.

\section{4 基于分子间作用纳机械敏感效应的生化 传感器}

\section{1 $\mathrm{SiO}_{2}$ 包覆的硅压阻悬臂梁、金表面修饰颈基敏 感基团与 ppb 痕量沙林传感器}

静态弯曲悬臂梁是一种表征分子特异性作用引 起纳机械表面应力的新型微纳敏感结构. 当悬臂梁 的一个表面在纳机械表面应力作用下发生弯曲时, 这种弯曲的机械响应可转化成电学信号读出. 最早 用于检测微悬臂梁弯曲的技术借鉴于 AFM 光学杜杆 检测系统, 是微悬臂梁传感器的传统检测方式. 光学 检测的最大优点在于其超高的灵敏度和相对简单的 检测平台. 通常, 直接采用分立的仪器式光学位移检 测器结合微悬臂梁, 就能够形成微悬臂梁传感系统, 这种检测方式在空气和溶液中(透明)都有很好的表
现. 但该检测方式的缺点在于: 微悬臂梁要特别设计 有足够光亮、足够大的光反射面, 整个系统需要构建 在光学减震设备上以减少干扰, 激光及监视设备体 积庞大且调节比较麻烦. 另外, 在浑浊的液体环境该 光检测方法很难实现. 为了克服这些缺点, 直接集成 自检测器件在微悬臂梁上近期成为公认的现场检测 方式. 硅是很好的压阻材料, 在悬臂梁上集成硅压阻 可直接检测悬臂梁的弯曲应力获得高灵敏度信号. 通过优化设计和制作工艺, 集成式力敏悬臂梁检测 已经达到了原子级的位移分辨率. 通过将压阻元件 连接成电桥而将其电阻值的变化转化成电压差动输 出, 压阻检测方式可以接近达到光学检测的灵敏度. 而且, 其最大的优势在于制作简单并与 MEMS 和 IC 工艺兼容, 因此集成压阻悬臂梁成为检测纳机械表 面应力传感器的近期研究热点, 可用来进行生化分 子识别和在线监测.

悬臂梁的机械响应灵敏度除了与所受的应力相 关之外, 还取决于悬臂梁的几何形状和材料本身的 性质. 图 10(a)示意了一种集成压阻 $\mathrm{SiO}_{2}$ 微悬臂梁器 件, 用来实现化学分子的超微量检测 ${ }^{[37]}$. 悬臂梁的主 体由 $\mathrm{SiO}_{2}$ 材料构成, 长 90 , 宽 21 , 厚 $1 \mu \mathrm{m}$. 单晶硅 压敏敏感电阻厚 $100 \mathrm{~nm}$, 硅上生长的 $\mathrm{SiO}_{2}$ 绝缘层厚 $80 \mathrm{~nm}$. 正如图 10(b)对所制造的微悬臂梁照片所示, 对于检测用微悬臂梁, 为了便于敏感分子层的选择 性固定, 在悬臂梁表面蒸镀了一层 $5 \mathrm{~nm} / 50 \mathrm{~nm}$ 厚度 的 $\mathrm{Cr} / \mathrm{Au}$ 薄膜.

在表面应力的作用下, 由于 $\mathrm{SiO}_{2}$ 的杨氏模量远 比硅的小, $\mathrm{SiO}_{2}$ 悬臂梁可以产生比传统的 $\mathrm{Si}$ 或 $\mathrm{SiN}_{x}$ 悬臂梁更大的弯曲灵敏度, 因此采用 $\mathrm{SiO}_{2}$ 制作悬臂 梁可以使器件具有更高的纳机械灵敏度. 另一方面, 由于硅压阻元件完全被 $\mathrm{SiO}_{2}$ 所包覆, 相比传统的硅 PN 结绝缘可以减少漏电噪声, 从而大大减小背景漂 移和噪声水平, 进一步提高检测分辨率. 从设计的悬 臂梁结构, 可以从理论上计算出悬臂梁的表面应力 敏感度为 $\sigma^{-1} \Delta R / R=8.37 \times 10^{-4}(\mathrm{~m} / \mathrm{N})$, 这其中 $\Delta R / R$ 是压阻的相对变化.

基于 SOI(绝缘体上的硅)材料, 采用图 10(c)列出 的硅微机械工艺流程, 制作出了集成压阻于梁上的 表面应力内检测传感器结构. 如图 10(d)所示, 在芯 片上共集成了 2 根悬臂梁即敏感梁和参考梁. 每根悬 臂梁上都集成了 1 个“几字”形的单晶硅压阻检测元件, 这种形状一方面可以保证形成的电阻值足够大，另 


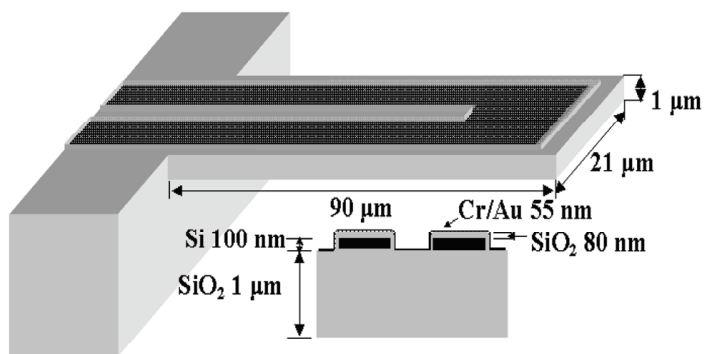

(a)

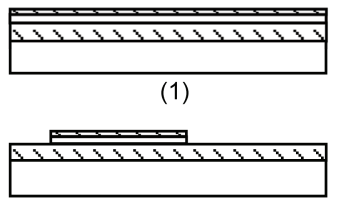

(2)

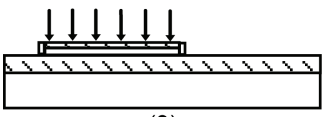

(3)
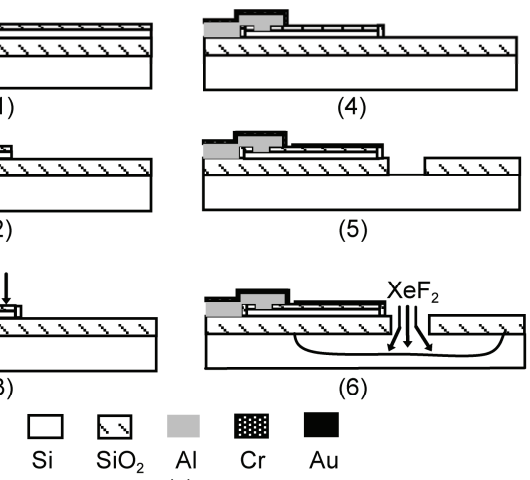

(5)

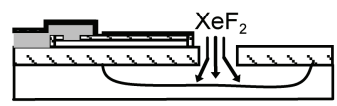

(6)

$\underset{\mathrm{Cr}}{\mathrm{Au}}$

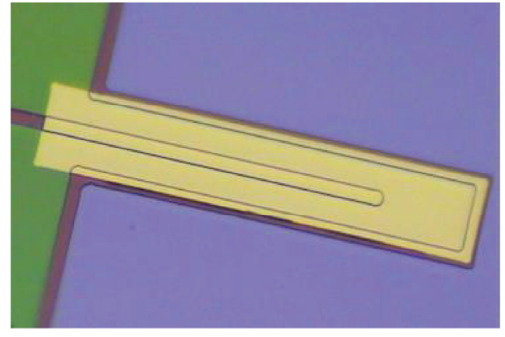

(b)

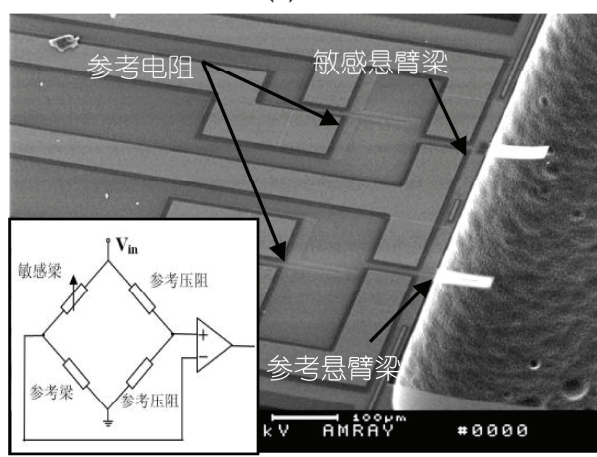

(d)

图 10 (网络版彩图) $\mathrm{SiO}_{2}$ 包覆的压阻微悬臂梁传感器

(a) 集成压阻悬臂梁的结构示意图; (b) 制成的敏感悬臂梁顶视照片, 梁表面蒸镀有金薄膜; (c) 悬臂梁的微机械制造工艺流程图; (d) 制 造的悬臂梁传感器 SEM 图像以及连接成惠斯通电桥的结构示意图 ${ }^{[20,36]}$

一方面可以使压阻沿悬臂梁长宽方向分布以检测微 悬臂梁的双轴弯曲应力. 其中敏感梁表面有金薄膜 覆盖以便于修饰巯基敏感分子层, 而参考梁上没有 金薄膜. 当微悬臂梁弯曲时, 相应会在压敏电阻中产 生应力变化, 由于压阻效应, 压敏电阻中的应力变化 将直接导致其的阻值变化. 将两个悬臂梁上的压阻 与在祄底上制作的 2 个电阻通过惠斯顿电桥方式进 行连接, 如图中的插图所示. 给电桥两端输入一定的 电压, 通过测量电压信号来表征压敏电阻的变化值. 图 10(d)的传感器结构包括一根正面有敏感膜(在正面 的金薄膜上生长)的敏感悬臂梁和另一根没有敏感层 的参考悬臂梁. 由于 2 根硅梁的主体结构相同, 具有 相近的温度特性, 可以利用惠斯通电桥的连接方式 来消除大部分环境温度变化造成的噪声信号, 如敏 感压阻元件的温度漂移系数等.

在上述硅压阻悬臂梁生化分子检测传感器的设 计和制造方面, 有如下因素需要着重考虑. 首先要保 证悬臂梁结构具有较小的残余应力以提高检测分辨 能力. 如在图 10 总所展示的包裹了单晶硅压阻层的 氧化硅悬臂梁结构, 需要使作为力学结构材料的氧 化硅厚度远大于作为压阻敏感层的单晶硅厚度, 才
能尽可能地降低两种材料热不匹配所带来的残余热 应力效应(即双金属效应). 反之, 当两种材料厚度相 近时, 温度变化造成的双金属效应会严重地影响检 测信号的稳定性. 在设计和制造中, 悬臂梁总的厚度 不能太厚, 反之悬臂梁表面分子间作用产生的较小 量值的表面应力将不会引起悬臂梁的较强烈弯曲, 以致使压阻检测的灵敏度过低. 当然, 为了保证压阻 敏感硅层有一定厚度, 以确保有足够的电桥检测电 流流过压阻元件, 悬臂梁的厚度减薄程度是受到限 制的. 为使传感器具有抗外界振动冲击干扰的可靠 性, 需要将悬臂梁的固有谐振频率设计到远离常见 环境振动频谱的区域，一般应在 $10 \mathrm{kHz}$ 以上.

以有机磷化合物为代表的神经性毒剂一直被认 为是目前最毒的化学毒剂, 沙林(甲氟膦酸异丙酯)是 其中的典型代表, 在极低浓度下就可以造成人员中 毒伤亡, 在 $10^{-3} \mathrm{mg} / \mathrm{L}$ 即 $200 \mathrm{ppb}$ 浓度下, 数分钟就可 以致人死亡. 因此, 它是目前化学反恐的主要目标化 合物之一. 沙林的超微量检测是一个急待解决的难 题，实验室通常采用甲基膦酸二甲酯(DMMP)作为沙 林的模剂进行研究. 沙林和 DMMP 的化学结构非常 相似, 它们都含有 $-\mathrm{P}=\mathrm{O}$ 和 $-\mathrm{P}-\mathrm{O}-\mathrm{C}$ 键, 但因 DMMP 的 
毒性很小, 实验室模拟检测时操作相对比较安全. 因 此, 研究经常采用 DMMP 作为沙林模拟剂开展研究, 获得可用于有机磷化合物检测的传感器.

传感器的识别能力主要决定于敏感层的结构. 针对有机磷化合物的结构特点和悬臂梁敏感结构的 特性, 在文献[37]的研究中设计了一种 $\mathrm{Cu}^{2+}$ 颈基十 一酸(11-MUA)双分子层来识别和捕获有机磷化合物. 由于 $\mathrm{Cu}^{2+}$ 离子与磷酰键有很好的配位作用, 可以形
成 $\mathrm{P}=\mathrm{O}-\mathrm{Cu}^{2+}$ 配位结构, 二者的作用力也属于中等作 用力，因此可以利用 $\mathrm{Cu}^{2+}$ 的配位作用来识别有机磷 化合物中的磷酰键 ${ }^{[38 \sim 40]}$. 以 DMMP 分子为例, 其与 $\mathrm{Cu}^{2+} / 11-\mathrm{MUA}$ 双分子层作用导致悬臂梁产生应变的 过程和机制如图 11(a)所示. 敏感微悬臂梁上 $\mathrm{Cu}^{2+} / 11$ MUA 双分子敏感层采用自组装和嫁接修饰方法获得, 其结构以及在悬臂梁 $\mathrm{Au}$ 基底上的形成过程和 DMMP 分子在 $\mathrm{Cu}^{2+} / 11-\mathrm{MUA}$ 层上吸附与解吸过程如图 11(b)

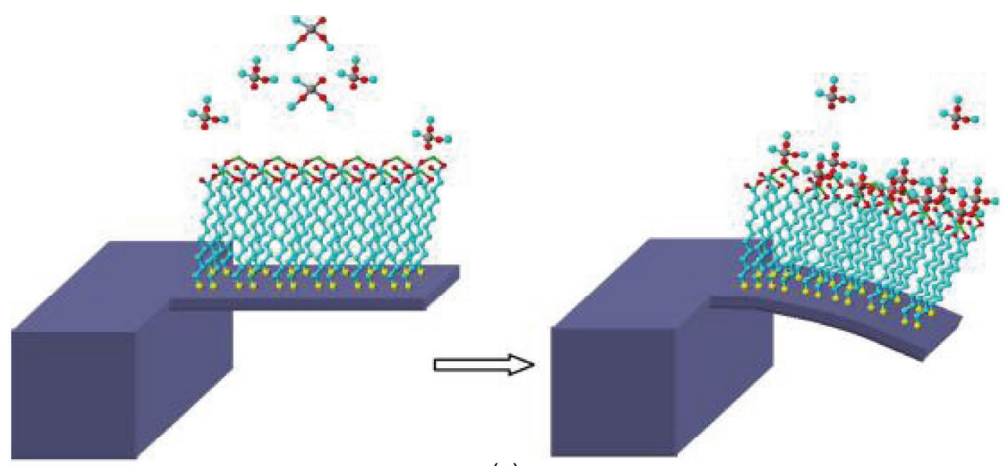

(a)
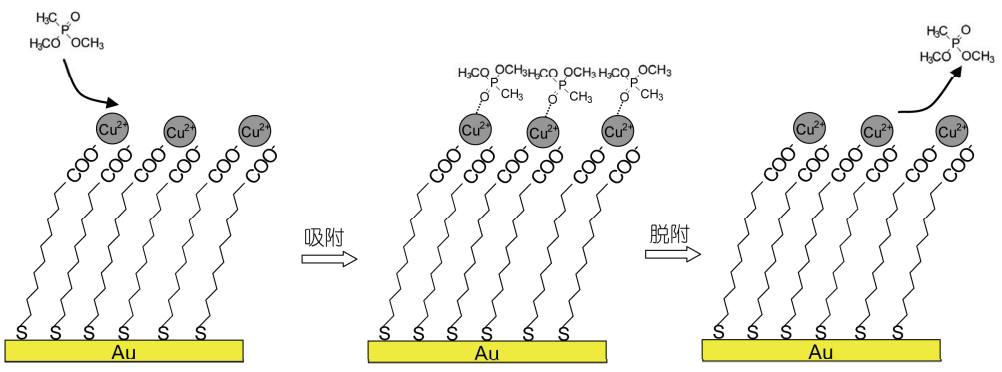

(b)

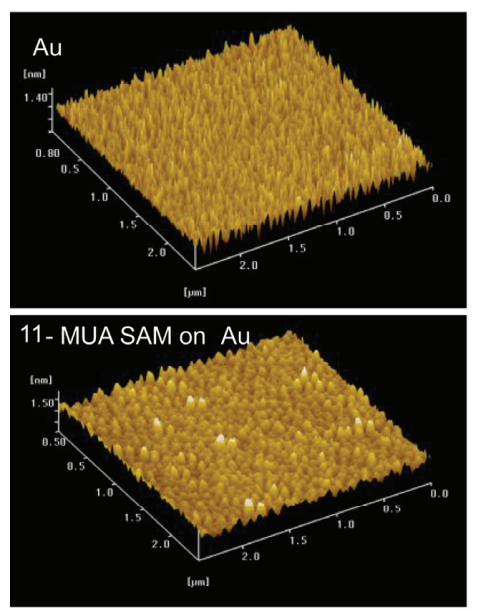

(c)

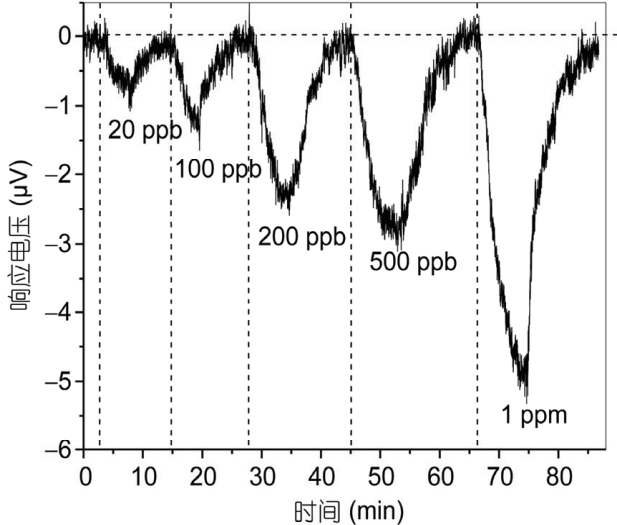

(d)

图 11 (网络版彩图) (a) DMMP 分子在 $\mathrm{Cu}^{2+} / 11-\mathrm{MUA}$ 双分子敏感层上特异性吸附产生表面压应力致使悬臂梁下弯的示意图; (b) DMMP 分子在双分子敏感层上吸附和解附的原理示意图; (c) 在金表面修饰 11-MUA 前后的表面 AFM 相貌差别; (d) 传 感器对 20 ppb 1 ppm 浓度 DMMP 实时检测的电桥差压输出信号 ${ }^{[37]}$ 
所示. 利用 AFM 和衰减全反射-傅里叶红外光谱 (ATR-FTIR)对自组装前后的表面进行了表征. 图 11(c)显示了 11-MUA 自组装前 $\mathrm{Au}$ 基底和自组装后 $\mathrm{SAM}$ 表面的 $\mathrm{AFM}$ 扫描图像. 从图中可以看出, 在 $\mathrm{Au}$ 表面组装 SAM 后, 表面趋于平坦化, 并呈现出颗粒 状结构. 从 11-MUA SAM 在 $\mathrm{Au}$ 表面生长后的 ART-FTIR 分析结果显示: 主要吸收峰与 11-MUA SAM 结构完全吻合. 表明利用此方法可以在悬臂梁 的 $\mathrm{Au}$ 的表面形成良好的 11-MUA $\mathrm{SAM}^{[39,40]}$. 此后, 铜离子在 11-MUA 上的嫁接也就取得了成功.

图 11(d)给出了传感器对不同 ppm 浓度 DMMP 蒸气的响应曲线. 当传感器暴露于 DMMP 氛围后, 立即产生负电压响应, 约 $8 \mathrm{~min}$ 响应信号趋于平稳. 再次用氮气吹扫检测池时, 传感器的响应电压又逐 渐恢复至基线位置. 传感器对 $20 \mathrm{ppb}\left(10^{-4} \mathrm{mg} / \mathrm{L}\right)$ 浓度 的 DMMP 都有着很明显的响应. 同时, 传感器的响 应信号随着 DMMP 浓度的变大而增强. 此外由输出 电压的基线可以看出, 传感器的噪声仅为 $0.3 \mu \mathrm{V}$, 这 表明压阻式悬臂梁传感器经 $\mathrm{SiO}_{2}$ 完全包覆绝缘后, 漏电噪声很低, 从而实现较高的分辨率. 假定传感器 的响应电压大小正比于被吸附的 DMMP 分子数, 基 于 Langmuir 吸附等温曲线模型可以估算出传感器对 DMMP 的最小检测浓度约为几 ppb.

\section{2 在悬臂梁 $\mathrm{SiO}_{2}$ 表面直接修饰硅烷基敏感基团 及痕量 TNT 爆炸物传感器 ${ }^{[36]}$}

在贵金属表面自组装的颈基 SAM 在生物传感器 方面得到了广泛应用, 但是颈基 SAM 在空气环境中 的稳定性决定着其是否能够得到长期可靠应用. Schoenfisch 和 Pemberton ${ }^{[41]}$, Scott 等人 ${ }^{[42]}$, Willy 等 人 ${ }^{[43]}$ 对于暴露在空气中的颈基 SAM 进行的研究表明: 統基在空气环境中会迅速地被氧化, 生成亚磺酸盐 以及磺酸盐, 进而会从金表面脱附. 计算表明 $\mathrm{Au}-\mathrm{S}$ 键能只有 $1.6 \mathrm{eV}^{[44,45]}$, 相当微弱. 在 $\mathrm{Au}-\mathrm{S}$ 键形成的 过程中, 每个 $\mathrm{Au}$ 原子上约有 $0.30 \mathrm{e}\left(0.48 \times 10^{-19} \mathrm{C}\right)$ 的 电荷转移至 $\mathrm{S}$ 原子上. 由此, 在 $\mathrm{Au}-\mathrm{S}$ 结合的界面上 将形成一层带有正电荷的 $\mathrm{Au}$ 原子层和带有负电荷的 $\mathrm{S}$ 原子层, 这将直接导致层内原子间的库仑排斥作用. 有关巯基化合物的研究表明, 颈基 SAM 在温度升高 到 $60^{\circ} \mathrm{C}$ 以上的条件下也会逐渐从 $\mathrm{Au}$ 表面解吸附 ${ }^{[46]}$, 说明在温度变化的环境下, 金表面的放基 SAM 不够 稳定. 以上原因都造成了統基 SAM 易于从金属表面
脱附. 当颈基 SAM 中部分分子从金表面脱附后, 失 去了致密排布的颈基分子之间的距离大大增加. 对 于检测爆炸物 TNT(三硝基甲苯)分子来说，可能只有 一个硝基结合在羧基头基上，另外的 1 个硝基悬空， 这样就无法产生分子间的排斥作用了. 此外, 在稀疏 分布的放基 SAM 上吸附的 TNT 分子之间由于无法紧 密分布, 也不会产生足够的横向作用效应敏感信号. 为了解决颈基敏感层稳定性的弱点, 在悬臂梁 $\mathrm{SiO}_{2}$ 表面直接修饰硅氧烷敏感膜的方法成为一个解决方 案, 原因是这样的敏感分子层采用的是稳定而可靠 的共价键结合.

对于 TNT 分子的检测，一般选取对于硝基具有 特异性敏感的氢键酸类化合物, 如羧酸. 已有研究证 明，这一类的化合物对于硝基类爆炸物的蒸气具有 良好的特异性 ${ }^{[8]}$, 因而可以用作敏感基团. 而硅氧烷 化合物一般是通过硅甲氧基与氧化硅表面的硅羟基 反应形成 $\mathrm{Si}-\mathrm{O}$ 键固定的. 如果能够合成一种能在氧 化硅表面固定的、又能够对于 TNT 分子敏感的化合 物, 就可以达到我们的设计目标. 但不幸的是, 满足 这两项要求的硅氧烷基团和羧基基团之间会自发地 发生缩聚反应. 缩聚反应后, 对 TNT 敏感的羧基将 不存在. 因此, 类似于統基敏感层在溶液中直接自组 装到悬臂梁表面的方法是不可行的, 因为缩聚反应 致使敏感基团无法真正形成在悬臂梁的表面. 基于 同样原因, 也很难通过化学合成的方法制备这种末 端是羧基的硅氧烷化合物.

为了解决这个问题, 文献[36]中报道的研究提出 了一种分两步合成的方法: 首先在 $\mathrm{SiO}_{2}$ 表面自组装 1 层硅氧烷化合物, 该化合物的尾端在一定的化学反 应条件在可以进行嫁接, 将第 2 层带有敏感头基的分 子连接到第 1 层硅氧烷自组装层上, 从而形成敏感双 层膜. 在构筑敏感双层膜的过程中, 嫁接过程需要控 制在严格条件下. 研究选择了尾端是环氧基团的硅 氧烷化合物. 由于环氧基团在无水条件下比较稳定, 遇水后易于水解, 反应条件的控制相对容易, 因此研 究选择了环氧基团作为分子层间嫁接平台. 其具体 过程如图 12 所示.

完成了硅氧烷自组装敏感双层膜在 $\mathrm{SiO}_{2}$ 悬臂梁 传感器的修饰之后, 用器件对 TNT 蒸气进行了敏感 检测试验. TNT 分子被悬臂梁表面敏感层上的羧基特 异性地吸附从而产生表面应力, 导致悬臂梁的弯曲, 输出压阻信号. 需要指出的是, 在这里的修饰过程中, 

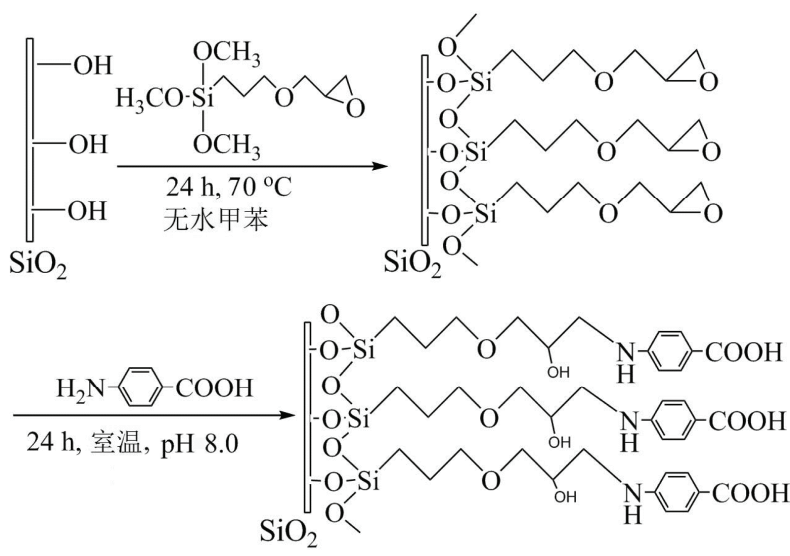

图 12 悬臂梁 $\mathrm{SiO}_{2}$ 表面上修饰硅氧烷自组装敏感双分子层 的示意图 ${ }^{[36]}$

硅氧烷自组装敏感双层膜修饰在敏感悬臂梁的 $\mathrm{SiO}_{2}$ 下表面, 而不是在敏感悬臂梁的 $\mathrm{Au}$ 表面进行修饰,
特异性吸附将会使悬臂梁产生弯曲. 而对于参考悬 臂梁, 其上下表面都是 $\mathrm{SiO}_{2}, 2$ 个表面上由于特异性 吸附导致的弯曲作用会相互抵消，不会造成悬臂梁 产生弯曲, 如图 13 所示.

传感器对于不同浓度 TNT 蒸气的敏感响应在图 14(a)中所示. 从图中可以看出, 传感器对 $0.1 \mathrm{ppb}$ 的 $\mathrm{TNT}$ 蒸气的响应非常明显, 有近 $4 \mu \mathrm{V}$. 对于 7.6 和 25 $\mathrm{ppb}$ 浓度的 TNT 分别有 $17,30 \mu \mathrm{V}$ 左右的响应信号. 根据信噪比可以估算, 传感器对于 TNT 蒸气的检测 浓度下限可以达到数十 $\operatorname{ppt}$ (十亿分之一)的水平. 为 了研究硅氧烷敏感双层膜的工作寿命, 我们使用同 一只传感器对于浓度为 $7.6 \mathrm{ppb}$ 的 TNT 蒸气进行了测 试, 并在 $140 \mathrm{~d}$ 之后重复测试相同浓度的 TNT 蒸气, 在这期间, 器件放置于培养血中, 并保存在普通实验 室的环境中. 结果如图 14(b)所示. 可以看出, 第一次 测试的响应信号约为 $17.2 \mu \mathrm{V}$, 第二次测试的响应信
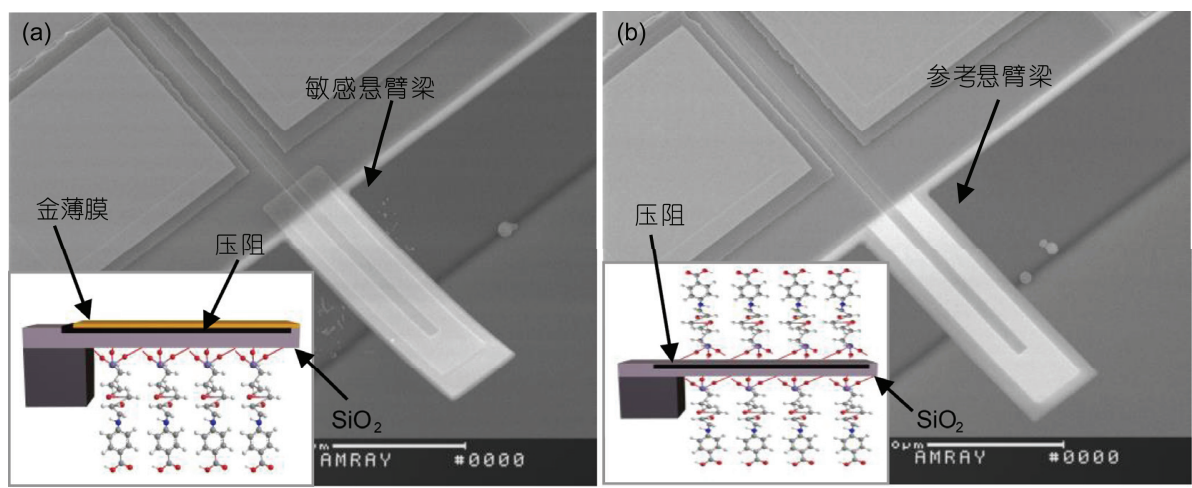

图 13 (网络版彩图)敏感悬臂梁(a)和参考悬臂梁(b)SEM 照片

(a) 插图为硅氧烷敏感双层膜修饰在悬臂梁下表面的示意图; (b) 插图为敏感膜修饰在悬臂梁上下两个表面的示意图 ${ }^{[36]}$

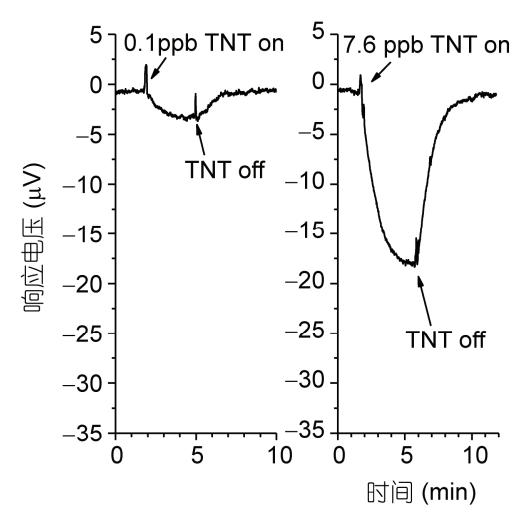

(a)
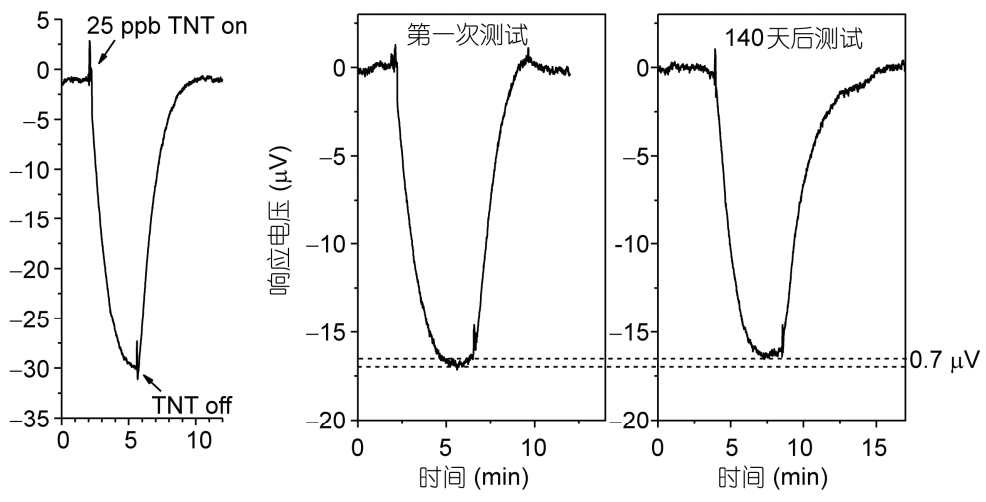

(b)

图 14 (a) 传感器对于不同浓度 TNT 蒸气的响应; (b) 同一只传感器对 $7.6 \mathrm{ppb}$ 浓度 TNT 的首次测试响应以及 $140 \mathrm{~d}$ 后再次 测试响应 ${ }^{[36]}$ 
号没有明显的衰减, 为 $16.5 \mu \mathrm{V}$, 响应过程保持了迅 速且可逆的优点, 证明了使用硅氧烷敏感双层膜修 饰的传感器具备较长的工作寿命. 相对于以往的颈 基 SAM 敏感修饰方法, 这种使用硅氧烷自组装敏感 双层膜修饰的新方法明显地改善了悬臂梁化学传感 器的长期工作可靠性和稳定性, 也使该研究的 TNT 传感器朝着实际应用前进了重要的一步.

\section{3 液相检测的悬臂梁生物传感器 ${ }^{[35]}$}

在传统悬臂梁生化传感器中, 最为常见的是利 用光学方法检测的悬臂梁, 通过检测入射到悬臂梁 自由端的激光束位置的微小偏移来检测悬臂梁的弯 曲. 然而, 这种光学检测方法会受到溶液透明度差和 溶液折射率变化等因素导致激光束检测精度受到影 响. 此外光学检测装置体积庞大, 使用过程中经常需 要进行光学校准, 操作复杂且耗时 ${ }^{[47]}$. 而文献[35]报 道的研究中制作了集成超薄单晶硅压敏电阻的 $\mathrm{SiO}_{2}$ 悬臂梁, 将悬臂梁的弯曲转换为电学信号输出, 避免 了上述在溶液中进行光学检测遇到的问题, 更适宜 在线检测在溶液中进行的反应, 而且检测电路简单 便宜.

对于集成有压阻的 $\mathrm{SiO}_{2}$ 悬臂梁来说, 尽管压阻 完全包裹在 $\mathrm{SiO}_{2}$ 绝缘层内, 但是压阻检测的信号需 要引线输出, 芯片表面必然排布着金属导线, 而生化 反应中的检测溶液多为导电性溶液, 会在溶液中造 成短路而无法检测. 为了解决这个问题，该研究在金 属引线上制作一层光敏的聚合物 SU-8 绝缘层进行屏 蔽, 仅仅在压焊盘处保留金属焊盘. 在敏感压焊封装 完成之后, 使用硅橡胶将其封闭, 这样可以使整个器 件的电路绝缘，以便于在溶液中进行检测，所制成的 可在液态环境中检测的悬臂梁传感器如图 15(a)所示.
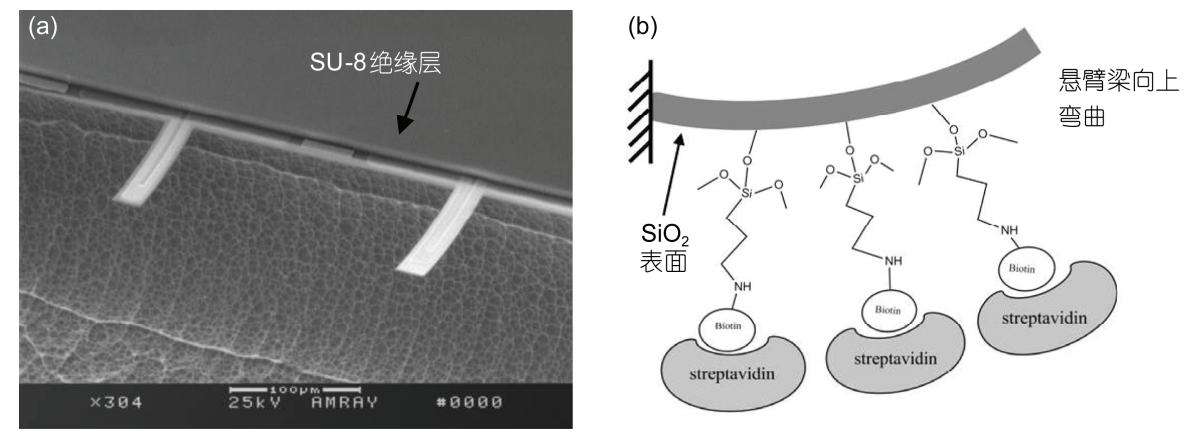

图 15 (a) 液相检测悬臂梁的 SEM 照片, 右侧为敏感悬臂梁, 左侧为参考悬臂梁, 金属引线被 SU-8 绝缘层所覆盖; (b) 在 $\mathrm{SiO}_{2}$ 表面固定生物素以及生物素-亲和素特异性结合在悬臂梁表面结合产生压应力使悬臂梁弯曲的示意 ${ }^{[3]}$ 

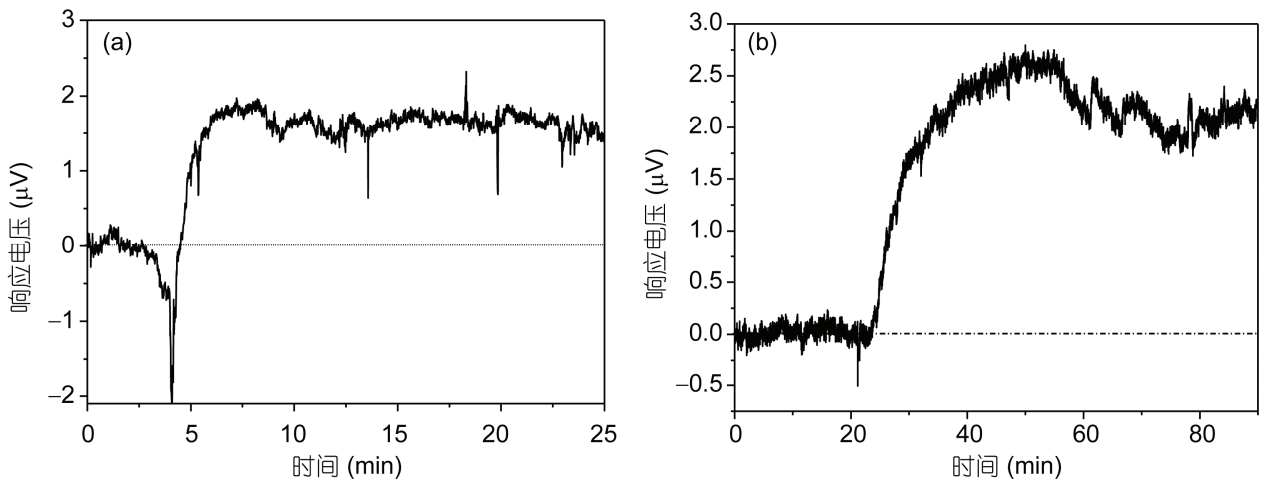

图 16 (a) 活化的生物素与悬臂梁表面的 APTES 硅氧烷敏感层结合的在线检测响应; (b) 链霉亲和素与悬臂梁上生物素的 特异性结合的在线检测响应 ${ }^{[35]}$

没, 待基线平稳后开始记录. 向检测池中注入活化的 生物素溶液(浓度为 $10^{-6} \mathrm{~mol} / \mathrm{mL}$ ), 实时记录半小时 内传感器的响应, 如图 16(a)所示. 活化的生物素与 APTES 通过酰胺反应结合到 $\mathrm{SiO}_{2}$ 悬臂梁表面, 响应 的压阻输出信号在 $2 \mu \mathrm{V}$ 左右, 可以清晰识别出悬臂 梁是向上弯曲, 表明表面受到了压应力.

在 $\mathrm{SiO}_{2}$ 悬臂梁表面固定了可以检测亲和素的探 针并用 BSA 封闭之后, 检测了荧光标记的链霉亲和 素. 先将传感器浸没于 PBS 溶液中, 待基线平稳后, 加入苂光标记的链霉亲和素并在线检测 1 小时. 如图 16 (b) 所示, 传感器对于浓度为 $1.5 \times 10^{-11} \mathrm{~mol} / \mathrm{mL}$ 的链 霉亲和素的响应信号约为 $2.5 \mu \mathrm{V}$. 特异性反应使得 悬臂梁向上弯曲. 测试完成后, 使用苂光显微镜观察 到了悬臂梁上有明显的绿色荧光, 证明了检测信号 确实来至于亲和素与生物素之间成功的结合作用.

\section{5 讨论与展望}

利用探针分子与目标探测分子间相互作用产生 的纳机械敏感效应来探测生化分子, 是一个很有前 途的研究方向. 基于该敏感原理的传感器结构如微 纳悬臂梁等将会像 AFM 探针一样在不远的将来取得 痕量分子探测方面突破性的进展, 最终实现所谓单 分子和单原子级的敏感探测水平. 为了更好地实现 这样的目标, 一方面要在现有基础上进一步建立分 子作用产生纳机械信号的定量化模型, 进而指导敏 感结构和材料的优化设计, 不断提高检测水平. 另一 方面, 采用 MEMS/NEMS 技术在未来会开发出越来 越敏感和越来越廉价的微纳传感器以及传感器集成 阵列, 实现在线的多通道痕量生化分子检测识别, 最 终真正广泛应用到生物医药、食品安全和环境保护等 重要领域中.

\section{参考文献}

1 Binnig G, Rohrer H. Scanning tunneling microscopy. Helv Phys Acta, 1982, 55: 726-735

2 Binnig G, Quate C, Gerber C. Atomic force microscope. Phys Rev Lett, 1986, 56: 930-933

3 Yang Z, Li Xinxin, Wang Y, et al. Micro cantilever probe array integrated with piezoresistive sensor. Microelectr J, 2004, 35: 479-483

4 Li X X, Lee D W. Integrated micro-cantilevers for ultra-resoluble sensing and probing. Meas Sci Technol, 2012, 23: 022001

5 Yu H T, Yang T T, Chen Y, et al. Chemo-mechanical Joint-detection with both dynamic and static micro-cantilevers for inter-homologue molecular identification. Anal Chem, 2012, 84: 6679-6685

6 Berger R, Delamarche E, Lang H, et al. Surface stress in the self-assembly of alkanethiols on gold. Science, 1997, 276: 2021-2024

7 Fritz J, Baller M, Lang H, et al. Translating biomolecular recognition into nanomechanics. Science, 2000, 288: 316-318

8 Senesac L, Thundat T. Nanosensors for trace explosive detection. Mater Today, 2008, 11: 28-36

9 Ji H, Finot E, Dabestani R, et al. A novel self-assembled monolayer (SAM) coated microcantilever for low level caesium detection. Chem Commun, 2000, 6: 457-458

10 Pinnaduwage L, Boiadjiev V, Hawk J, et al. Sensitive detection of plastic explosives with self-assembled monolayer-coated 
microcantilevers. Appl Phys Lett, 2003, 83: 1471-1473

11 Ono T, Li X X, Miyashita H, Esashi M. Mass sensing of adsorbed molecules in sub-picogram sample with ultrathin silicon resonator. Rev Sci Instrum. 2003, 74: 1240-1243

12 Hanay M, Kelber S, Naik A, et al. Single-protein nanomechanical mass spectrometry in real time. Nat Nanotechnol, 2012, 7: 602-608

13 Arlett J L, Myers E B, Roukes M L. Comparative advantages of mechanical biosensors. Nat Nanotechnol, 2011, 6: 203-215

14 Yang Y, Callegari C, Feng X, et al. Zeptogram-scale nanomechanical mass sensing. Nano Lett, 2006, 6: 583-586

15 McCaig H C, Myers E, Lewis N S, et al. Vapor sensing characteristics of nanoelectromechanical chemical sensors functionalized using surface-initiated polymerization. Nano Lett, 2014, 14: 3728-3732

16 Chaste J, Eichler A, Moser J, et al. A nanomechanical mass sensor with yoctogram resolution. Nat Nanotechnol, 2012, 7: 301-304

17 Godin M, Tabard-Cossa V, Miyahara Y, et al. Cantilever-based sensing: The origin of surface stress and optimization strategies. Nanotechnology, 2010, 21: 075501

18 Godin M, Williams P, Tabard-Cossa V, et al. Surface stress, kinetics, and structure of alkanethiol self-assembled monolayers. Langmuir, 2004, 20: 7090-7096

19 Stoney G. The tension of metallic films deposited by electrolysis. Proceedings of the Royal Society of London. Series A, Containing Papers of a Mathematical and Physical Character. Vol 82. Edinburgh: The Royal Society, 1909. 172-175

20 Li P, Li X X. A single-sided micromachined piezoresistive $\mathrm{SiO}_{2}$ cantilever sensor for ultra-sensitive detection of gaseous chemicals. J Micromech Microeng, 2006, 16: 2539-2546

21 Li P, Li X X, Zuo G, et al. Silicon dioxide microcantilever with piezoresistive element integrated for portable ultraresoluble gaseous detection. Appl Phys Lett, 2006, 89: 074104

22 Desikan R, Lee I, Thundat T. Effect of nanometer surface morphology on surface stress and adsorption kinetics of alkanethiol self-assembled monolayers. Ultramicroscopy, 2006, 106: 795-799

23 Zhang F. Fundamentals of Molecular Interface Chemistry. Shanghai: Shanghai science and technology literature press, 2006

24 Adamson A, Gast A. Physical Chemistry of Surfaces. 6th ed. New York: John Wiley \& Son, 1997

25 Zuo G, Li X X. A three-layer model of self-assembly induced surface-energy variation experimentally extracted by using nanomechanically sensitive cantilevers. Nanotehcnology, 2011, 22: 045501

26 Yang T, Li X X, Chen Y, et al. Adsorption induced surface-stress sensing signal originated from both vertical interface effects and intermolecular lateral interactions. Analyst, 2011, 136: 5261-5269

$27 \mathrm{Wu} \mathrm{G}$, Ji H, Hansen K, et al. Origin of nanomechanical cantilever motion generated from biomolecular interactions. Proc Natl Acad Sci USA, 2001, 98: 1560-1564

28 Love J C, Estroff L A., Kriebel J K, et al. Self-assembled monolayers of thiolates on metals as a form of nanotechnology. Chem Rev, 2005, 105: 1103-1169

29 Ulman A. Formation and structure of self-assembled monolayers. Chem Rev, 1996, 96: 1533-1554

30 Laibinis P E, Whitesides G M, Allara D L, et al. Comparison of the structures and wetting properties of self-assembled monolayers of $n$-alkanethiols on the coinage metal surfaces, copper, silver, and gold. J Am Chem Soc, 1991, 113: 7152-7167

31 Feng X, Fryxell G E, Wang L Q, et al. Functionalized monolayers on ordered mesoporous supports. Science, 1997, 276: 923-926

32 Wight A P, Davis M E. Design and preparation of organic-inorganic hybrid catalysts. Chem Rev, 2002, 102: 3589-3613

33 Dareing D W, Thundat T. Simulation of adsorption-induced stress of a microcantilever sensor. J Appl Phys, 2005, 97: 043526

34 Sushko M L, Harding J H, Shluger A L, et al. Physics of Nanomechanical biosensing on cantilever arrays. Adv Mater, 2008, 20: 3848-3853

35 Chen Y, Xu P C, Liu M, et al. Bio/chemical detection in liquid with self-sensing Pr-Oxi-Lever (piezo-resistive $\mathrm{SiO}_{2}$ cantilever) sensors. Microelectron Eng, 2010, 87: 2468-2474

36 Chen Y, Xu P, Li X X. Self-assembling siloxane bilayer directly on $\mathrm{SiO}_{2}$ surface of micro-cantilevers for long-term highly repeatable sensing to trace explosives. Nanotechnology, 2010, 21: 265501

37 Zuo G, Li X X, Li P, et al. Detection of trace organophosphorus vapor with a self-assembled bilayer functionalized $\mathrm{SiO}_{2}$ microcantilever piezoresistive sensor. Anal Chim Acta, 2006, 580: 123-127

38 Crooks R, Ricco A. New organic materials suitable for use in chemical sensor arrays. Acc Chem Res, 1998, 31: 219-227

39 Kepley L, Crooks R, Ricco A. Selective surface acoustic wave-based organophosphonate dhemical sensor employing a self-assembled composite monolayer: A new paradigm for sensor design. Anal Chem, 1992, 64: 3191-3193

40 Crooks R, Yang H, McEllistrem L, et al. Interactions between self-assembled monolayers and an organophosphonate. Faraday Discuss, 1997, 107: 285-305 
41 Schoenfisch M H, Pemberton J E. Air stability of alkanethiol self-assembled monolayers on silver and gold surfaces. J Am Chem Soc, 1998, 120: $4502-4513$

42 Scott J R, Baker L S, Everett W R, et al. Laser desorption fourier transform mass spectrometry exchange studies of air-oxidized alkanethiol self-assembled monolayers on gold. Anal Chem, 1997, 69: 2636-2639

43 Willey T M, Vance A L, van Buuren T, et al. Rapid degradation of alkanethiol-based self-assembled monolayers on gold in ambient laboratory conditions. Surf Sci, 2005, 576: 188-196

44 Ulman A. Formation and structure of self-assembled monolayers. Chem Rev, 1996, 96: 1533-1554

45 Dubois L H, Nuzzo R G. Synthesis, structure, and properties of model organic-surfaces. Annu Rev Phys Chem, 1992, 43: 437-463

46 Xiao X D, Wang B, Zhang C, et al. Thermal annealing effect of alkanethiol monolayers on Au(111) in air. Surf Sci, 2001, 472: 41-50

47 Lavrik N V, Sepaniak M J, Datskos P G. Cantilever transducers as a platform for chemical and biological sensors. Rev Sci Instrum, 2004, 75: 2229-2253

48 Wilchek M, Bayer E A. The avidin-biotin complex in bioanalytical applications. Anal Biochem, 1988, 171: 1-32

49 Zhao W, Xu J J, Chen H Y. Electrochemical biosensors based on layer-by-layer assemblies. Electroanal, 2006, 18: 1737-1748

\title{
Sensing effects and trace-level bio/chemical sensors based on molecular interaction induced nano-mechanical force
}

\author{
LI XinXin
}

State Key Lab of Transducer Technology, Shanghai Institute of Microsystem and Information Technology, Shanghai 200050, China

Surface Gibbs free energy can be changed by specific interaction between the targeted molecules and the functionalized molecule-layers pre-modified on MEMS/NEMS sensing structures. The generated nanomachining surface-stress can be electrically read out by the sensing element integrated on the MEMS/NEMS device. To explore the surface-stress generating mechanism that is originated from SAM (self-assembled monolayer) growth, we individually study on the three parts of head-group, molecule chain and tail-group. Based on the analysis of individual contribution from each part to surface energy change, a plotting method is developed to quantitatively assess and evaluate the effect of the grown SAM on surface energy change, i.e., generated surface-stress. In order to apply the nanomachining sensing effect into rapid biochemical detection and recognition, for the first time, we separately investigate the sensing effect from vertical specific interaction and lateral interaction between adjacent molecules. Following conclusion is finally declared based on theoretical analysis and experiment: the sign and the magnitude of the surface-stress are mainly determined by the lateral effect, while the vertical effect can make the molecule layer into ordered status thereby helping to the lateral effect. Besides, among various lateral molecule interaction, hydrogen-bond effect is the most effective one to generate the most significant sensing signal. Based on the nanomachining sensing mechanism, micro/nano cantilever sensors have been recently developed for rapid detection of trace-level biochemical molecules, which translate the surface-stress into cantilever bending and read out electric sensing signal by integrated piezoresitors. By modifying double-layer sensing molecules on gold surface of the cantilever, ppb-level organophorous agent is detected. To improve long-term stability of the sensing layer for TNT detection, a double-layer grafting technique is developed to directly modify siloxane sensing-groups on silicon surface of cantilever, thereby realizing long-term reliable detection to ppt-level TNT explosive. Finally, a SU8 selectively coated cantilever is developed for biological detection in liquid, with which $1.5 \times 10^{-11} \mathrm{~mol} / \mathrm{mL}$ streptavidin is real-time detected in solution.

specific molecule interaction, nano-mechanical sensing effect, surface stress, bio/chemical sensors

doi: 10.1360/N092014-00361 